\title{
Regularization with Metric Double Integrals of Functions with Values in a Set of Vectors
}

\author{
René Ciak ${ }^{1} \cdot$ Melanie Melching ${ }^{1}\left[0 \cdot\right.$ Otmar Scherzer $^{1,2}$
}

Received: 29 June 2018 / Accepted: 22 December 2018 / Published online: 7 February 2019

(c) The Author(s) 2019

\begin{abstract}
We present an approach for variational regularization of inverse and imaging problems for recovering functions with values in a set of vectors. We introduce regularization functionals, which are derivative-free double integrals of such functions. These regularization functionals are motivated from double integrals, which approximate Sobolev semi-norms of intensity functions. These were introduced in Bourgain et al. (Another look at Sobolev spaces. In: Menaldi, Rofman, Sulem (eds) Optimal control and partial differential equations-innovations and applications: in honor of professor Alain Bensoussan's 60th anniversary, IOS Press, Amsterdam, pp 439-455, 2001). For the proposed regularization functionals, we prove existence of minimizers as well as a stability and convergence result for functions with values in a set of vectors.
\end{abstract}

Keywords Regularization · Manifold-valued data $\cdot$ Non-convex $\cdot$ Metric $\cdot$ Double integral $\cdot$ Fractional Sobolev space Bounded variation

\section{Introduction}

Functions with values in a (nonlinear) subset of a vector space appear in several applications of imaging and in inverse problems, e.g.,

- Interferometric Synthetic Aperture Radar (InSAR) is a technique used in remote sensing and geodesy to generate, for example, digital elevation maps of the earth's surface. InSAR images represent phase differences of waves between two or more SAR images, cf. [44,53]. Therefore, InSAR data are functions $f: \Omega \rightarrow \mathbb{S}^{1} \subseteq \mathbb{R}^{2}$. The pointwise function values are on the $\mathbb{S}^{1}$, which is considered embedded into $\mathbb{R}^{2}$.

- A color image can be represented as a function in $H S V$ space (hue, saturation, value) (see, e.g., [48]). Color

Melanie Melching

melanie.melching@univie.ac.at

René Ciak

rene.ciak@univie.ac.at

Otmar Scherzer

otmar.scherzer@univie.ac.at

1 Computational Science Center, University of Vienna, Oskar-Morgenstern-Platz 1, 1090 Vienna, Austria

2 Johann Radon Institute for Computational and Applied Mathematics (RICAM), Altenbergstraße 69, 4040 Linz, Austria images are then described as functions $f: \Omega \rightarrow K \subseteq$ $\mathbb{R}^{3}$. Here $\Omega$ is a plane in $\mathbb{R}^{2}$, the image domain, and $K$ (representing the HSV space) is a cone in threedimensional space $\mathbb{R}^{3}$.

- Estimation of the foliage angle distribution has been considered, for instance, in [39,51]. Therefore, the imaging function is from $\Omega \subset \mathbb{R}^{2}$, a part of the Earth's surface, into $\mathbb{S}^{2} \subseteq \mathbb{R}^{3}$, representing foliage angle orientation.

- Estimation of functions with values in $S O(3) \subseteq \mathbb{R}^{3 \times 3}$. Such problems appear in Cryo-Electron Microscopy (see, for instance, $[38,58,61])$.

We emphasize that we are analyzing vector-, matrix-, tensor-valued functions, where pointwise function evaluations belong to some given (sub)set, but are always elements of the underlying vector space. This should not be confused with set-valued functions, where every function evaluation can be a set.

Inverse problems and imaging tasks, such as the ones mentioned above, might be unstable, or even worse, the solution could be ambiguous. Therefore, numerical algorithms for imaging need to be regularizing to obtain approximations of the desired solution in a stable manner. Consider the operator equation

$\mathrm{F}(w)=v^{0}$, 
where we assume that only (noisy) measurement data $v^{\delta}$ of $v^{0}$ become available. In this paper the method of choice is variational regularization which consists in calculating a minimizer of the variational regularization functional

$$
\mathcal{F}(w):=\mathcal{D}\left(\mathrm{F}(w), v^{\delta}\right)+\alpha \mathcal{R}(w) .
$$

Here

- $w$ is an element of the set of admissible functions.

- $\mathrm{F}$ is an operator modeling the image formation process (except the noise).

- $\mathcal{D}$ is called the data or fidelity term, which is used to compare a pair of data in the image domain, that is to quantify the difference of the two data sets.

- $\mathcal{R}$ is called regularization functional, which is used to impose certain properties onto a minimizer of the regularization functional $\mathcal{F}$.

- $\alpha>0$ is called regularization parameter and provides a trade off between stability and approximation properties of the minimizer of the regularization functional $\mathcal{F}$.

- $v^{\delta}$ denotes measurement data, which we consider noisy.

- $v^{0}$ denotes the exact data, which we assume to be not necessarily available.

The main objective of this paper is to introduce a general class of regularization functionals for functions with values in a set of vectors. In order to motivate our proposed class of regularization functionals, we review a class of regularization functionals appropriate for analyzing intensity data.

\subsection{Variational Regularization for Reconstruction of Intensity Data}

Opposite to what we consider in the present paper, most commonly, imaging data $v$ and admissible functions $w$, respectively, are considered to be representable as intensity functions. That is, they are functions from some subset $\Omega$ of an Euclidean space with real values.

In such a situation, the most widely used regularization functionals use regularization terms consisting of powers of Sobolev (see $[12,15,16]$ ) or total variation semi-norms [54]. It is common to speak about Tikhonov regularization (see, for instance, [59]) when the data term and the regularization functional are squared Hilbert space norms, respectively. For the Rudin, Osher, Fatemi (ROF) regularization [54], also known as total variation regularization, the data term is the squared $L^{2}$-norm and $\mathcal{R}(w)=|w|_{T V}$ is the total variation semi-norm. Nonlocal regularization operators based on the generalized nonlocal gradient are used in [35].

Other widely used regularization functionals are sparsity promoting [22,41], Besov space norms [42,46] and anisotropic regularization norms $[47,56]$. Aside from various regularization terms, there also have been proposed different fidelity terms other than quadratic norm fidelities, like the $p$ th powers of $\ell^{p}$ and $L^{p}$-norms of the differences of $F(w)$ and $v$, [55,57], maximum entropy [26,28] and Kullback-Leibler divergence [52] (see [50] for some reference work).

Our work utilizes results from the seminal paper of Bourgain, Brézis and Mironescu [14], which provides an equivalent derivative-free characterization of Sobolev spaces and the space $B V\left(\Omega, \mathbb{R}^{M}\right)$, the space of functions of bounded total variation, which consequently, in this context, was analyzed in Dávila and Ponce [23,49], respectively. It is shown in [14, Theorems 2 and 3'] and [23, Theorem 1] that when $\left(\rho_{\varepsilon}\right)_{\varepsilon>0}$ is a suitable sequence of nonnegative, radially symmetric, radially decreasing mollifiers, then

$$
\begin{aligned}
\lim _{\varepsilon \searrow 0} \tilde{\mathcal{R}}_{\varepsilon}(w) & :=\lim _{\varepsilon \searrow 0} \int_{\Omega \times \Omega} \frac{\|w(x)-w(y)\|_{\mathbb{R}}^{p}}{\|x-y\|_{\mathbb{R}^{N}}^{p}} \rho_{\varepsilon}(x-y) \mathrm{d}(x, y) \\
& = \begin{cases}C_{p, N}|w|_{W^{1, p}}^{p} & \text { if } w \in W^{1, p}(\Omega, \mathbb{R}), 1<p<\infty, \\
C_{1, N}|w|_{T V} & \text { if } w \in B V(\Omega, \mathbb{R}), p=1, \\
\infty & \text { otherwise, }\end{cases}
\end{aligned}
$$

Hence, $\tilde{\mathcal{R}}_{\varepsilon}$ approximates powers of Sobolev semi-norms and the total variation semi-norm, respectively. Variational imaging, consisting in minimization of $\mathcal{F}$ from Eq. 1.2 with $\mathcal{R}$ replaced by $\tilde{\mathcal{R}}_{\varepsilon}$, has been considered in $[3,11]$.

\subsection{Regularization of Functions with Values in a Set of Vectors}

In this paper we generalize the derivative-free characterization of Sobolev spaces and functions of bounded variation to functions $u: \Omega \rightarrow K$, where $K$ is some set of vectors, and use these functionals for variational regularization. The applications we have in mind contain that $K$ is a closed subset of $\mathbb{R}^{M}$ (for instance, HSV data) with nonzero measure, or that $K$ is a submanifold (for instance, InSAR data).

The reconstruction of manifold-valued data with variational regularization methods has already been subject to intensive research (see, for instance, [4,17-19,40,62]). The variational approaches mentioned above use regularization and fidelity functionals based on Sobolev and TV seminorms: a total variation regularizer for cyclic data on $\mathbb{S}^{1}$ was introduced in $[18,19]$, see also $[7,9,10]$. In $[4,6]$ combined first- and second-order differences and derivatives were used for regularization to restore manifold-valued data. The later mentioned papers, however, are formulated in a finite-dimensional setting, opposed to ours, which is considered in an infinite-dimensional setting. Algorithms for total variation minimization problems, including half-quadratic minimization and nonlocal patch-based methods, are given, for example, in $[4,5,8]$ as well as in $[37,43]$. On the theo- 
retical side the total variation of functions with values in a manifold was investigated by Giaquinta and Mucci using the theory of Cartesian currents in [33,34], and earlier [32] if the manifold is $\mathbb{S}^{1}$.

\subsection{Content and Particular Achievements of the Paper}

The contribution of this paper is to introduce and analytically analyze double integral regularization functionals for reconstructing functions with values in a set of vectors, generalizing functionals of the form Eq. 1.3. Moreover, we develop and analyze fidelity terms for comparing manifold-valued data. Summing these two terms provides a new class of regularization functionals of the form Eq. 1.2 for reconstructing manifold-valued data.

When analyzing our functionals, we encounter several differences to existing regularization theory (compare Sect. 2):

(i) The admissible functions, where we minimize the regularization functional on, do form only a set but not a linear space. As a consequence, well-posedness of the variational method (that is, existence of a minimizer of the energy functional) cannot directly be proven by applying standard direct methods in the Calculus of Variations [20,21].

(ii) The regularization functionals are defined via metrics and not norms, see Sect. 3 .

(iii) In general, the fidelity terms are non-convex. Stability and convergence results are proven in Sect. 4.

The model is validated in Sect. 6 where we present numerical results for denoising and inpainting of data of InSAR type.

\section{Setting}

In the following we introduce the basic notation and the set of admissible functions which we are regularizing on.

Assumption 2.1 All along this paper, we assume that

- $p_{1}, p_{2} \in[1,+\infty), s \in(0,1]$,

- $\Omega_{1}, \Omega_{2} \subseteq \mathbb{R}^{N}$ are nonempty, bounded and connected open sets with Lipschitz boundary, respectively,

- $k \in[0, N]$,

- $K_{1} \subseteq \mathbb{R}^{M_{1}}, K_{2} \subseteq \mathbb{R}^{M_{2}}$ are nonempty and closed subsets of $\mathbb{R}^{M_{1}}$ and $\mathbb{R}^{M_{2}}$, respectively.

Moreover,

- $\|\cdot\|_{\mathbb{R}^{N}}$ and $\|\cdot\|_{\mathbb{R}^{M_{i}}}, i=1,2$, are the Euclidean norms on $\mathbb{R}^{N}$ and $\mathbb{R}^{M_{i}}$, respectively.
- $\mathrm{d}_{\mathbb{R}^{M_{i}}}: \mathbb{R}^{M_{i}} \times \mathbb{R}^{M_{i}} \rightarrow[0,+\infty)$ denotes the Euclidean distance on $\mathbb{R}^{M_{i}}$ for $i=1,2$ and

- $\mathrm{d}_{i}:=\mathrm{d}_{K_{i}}: K_{i} \times K_{i} \rightarrow[0,+\infty)$ denote arbitrary metrics on $K_{i}$, which fulfill for $i=1$ and $i=2$

$-\left.\mathrm{d}_{\mathbb{R}^{M_{i}}}\right|_{K_{i} \times K_{i}} \leq d_{i}$,

- $\mathrm{d}_{i}$ is continuous with respect to $\left.\mathrm{d}_{\mathbb{R}^{M_{i}}}\right|_{K_{i} \times K_{i}}$, meaning that for a sequence $\left(a_{n}\right)_{n \in \mathbb{N}}$ in $K_{i} \subseteq \mathbb{R}^{M_{i}}$ converging to some $a \in K_{i}$ we also have $\mathrm{d}_{i}\left(a_{n}, a\right) \rightarrow 0$.

In particular, this assumption is valid if the metric $d_{i}$ is equivalent to $\left.\mathrm{d}_{\mathbb{R}^{M_{i}}}\right|_{K_{i} \times K_{i}}$. When the set $K_{i}, i=1,2$, is a suitable complete submanifold of $\mathbb{R}^{M_{i}}$, it seems natural to choose $d_{i}$ as the geodesic distance on the respective submanifolds.

- $\left(\rho_{\varepsilon}\right)_{\varepsilon>0}$ is a Dirac family of nonnegative, radially symmetric mollifiers, i.e., for every $\varepsilon>0$ we have

(i) $\rho_{\varepsilon} \in \mathcal{C}_{c}^{\infty}\left(\mathbb{R}^{N}, \mathbb{R}\right)$ is radially symmetric,

(ii) $\rho_{\varepsilon} \geq 0$,

(iii) $\int_{\mathbb{R}^{N}} \rho_{\varepsilon}(x) \mathrm{d} x=1$, and

(iv) for all $\delta>0, \lim _{\varepsilon \searrow 0} \int_{\left\{\|y\|_{\mathbb{R}^{N}}>\delta\right\}} \rho_{\varepsilon}(y) \mathrm{d} y=0$.

We demand further that, for every $\varepsilon>0$,

(v) there exists a $\tau>0$ and $\eta_{\tau}>0$ such that $\left\{z \in \mathbb{R}^{N}\right.$ : $\left.\rho_{\varepsilon}(z) \geq \tau\right\}=\left\{z \in \mathbb{R}^{N}:\|z\|_{\mathbb{R}^{N}} \leq \eta_{\tau}\right\}$.

This condition holds, e.g., if $\rho_{\varepsilon}$ is a radially decreasing continuous function with $\rho_{\varepsilon}(0)>0$.

- When we write $p, \Omega, K, M$, then we mean $p_{i}, \Omega_{i}, K_{i}$, $M_{i}$, for either $i=1,2$. In the following we will often omit the subscript indices whenever possible.

Example 2.2 Let $\hat{\rho} \in C_{c}^{\infty}\left(\mathbb{R}, \mathbb{R}_{+}\right)$be symmetric at 0 , monotonically decreasing on $[0, \infty)$ and satisfy

$\left|\mathbb{S}^{N-1}\right| \int_{0}^{\infty} \hat{t}^{N-1} \hat{\rho}(\hat{t}) \mathrm{d} \hat{t}=1$.

Defining mappings $\rho_{\varepsilon}: \mathbb{R}^{N} \rightarrow \mathbb{R}$ by

$\rho_{\varepsilon}(x):=\frac{1}{\varepsilon^{N}} \hat{\rho}\left(\frac{\|x\|_{\mathbb{R}^{N}}}{\varepsilon}\right)$

constitutes then a family $\left(\rho_{\varepsilon}\right)_{\varepsilon>0}$ which fulfills the above properties (i)-(v). Note here that

- by substitution $x=t \theta$ with $t>0, \theta \in \mathbb{S}^{N-1}$ and $\hat{t}=\frac{t}{\varepsilon}$,

$$
\begin{aligned}
\int_{\mathbb{R}^{N}} \rho_{\varepsilon}(x) \mathrm{d} x & =\frac{1}{\varepsilon^{N}} \int_{\mathbb{R}^{N}} \hat{\rho}\left(\frac{\|x\|_{\mathbb{R}^{N}}}{\varepsilon}\right) \mathrm{d} x \\
& =\frac{1}{\varepsilon^{N}} \int_{0}^{\infty} t^{N-1} \hat{\rho}\left(\frac{t}{\varepsilon}\right) \mathrm{d} t \int_{\mathbb{S}^{N-1}} \mathrm{~d} \theta \\
& =\left|\mathbb{S}^{N-1}\right| \int_{0}^{\infty} \hat{t}^{N-1} \hat{\rho}(\hat{t}) \mathrm{d} \hat{t}=1 .
\end{aligned}
$$


Here, $d \theta$ refers to the canonical spherical measure.

- Again by the same substitutions, taking into account that $\hat{\rho}$ has compact support, it follows for $\varepsilon>0$ sufficiently small that

$$
\begin{aligned}
\int_{\left\{y:\|y\|_{\left.\mathbb{R}^{N}>\delta\right\}}\right.} \rho_{\varepsilon}(x) \mathrm{d} x & =\frac{1}{\varepsilon^{N}} \int_{\left\{y:\|y\|_{\left.\mathbb{R}^{N}>\delta\right\}}\right.} \hat{\rho}\left(\frac{\|x\|_{\mathbb{R}^{N}}}{\varepsilon}\right) \mathrm{d} x \\
& =\frac{1}{\varepsilon^{N}} \int_{\delta}^{\infty} t^{N-1} \hat{\rho}\left(\frac{t}{\varepsilon}\right) \mathrm{d} t \int_{\mathbb{S}^{N-1}} \mathrm{~d} \theta \\
& =\left|\mathbb{S}^{N-1}\right| \int_{\delta / \varepsilon}^{\infty} \hat{t}^{N-1} \hat{\rho}(\hat{t}) \mathrm{d} \hat{t}=0 .
\end{aligned}
$$

In the following we write down the basic spaces and sets, which will be used in the course of the paper.

Definition 2.3 - The Lebesgue-Bochner space of $\mathbb{R}^{M_{-}}$ valued functions on $\Omega$ consists of the set

$$
L^{p}\left(\Omega, \mathbb{R}^{M}\right):=\left\{\phi: \Omega \rightarrow \mathbb{R}^{M}:\right.
$$

$\phi$ is Lebesgue-Borel measurable and

$$
\left.\|\phi(\cdot)\|_{\mathbb{R}^{M}}^{p}: \Omega \rightarrow \mathbb{R} \text { is Lebesgue-integrable on } \Omega\right\},
$$

which is associated with the norm $\|\cdot\|_{L^{p}\left(\Omega, \mathbb{R}^{M}\right)}$, given by

$$
\|\phi\|_{L^{p}\left(\Omega, \mathbb{R}^{M}\right)}:=\left(\int_{\Omega}\|\phi(x)\|_{\mathbb{R}^{M}}^{p} \mathrm{~d} x\right)^{1 / p} .
$$

- Let $0<s<1$. Then the fractional Sobolev space of order $s$ can be defined (cf. [1]) as the set

$$
\begin{aligned}
& W^{s, p}\left(\Omega, \mathbb{R}^{M}\right) \\
& :=\left\{w \in L^{p}\left(\Omega, \mathbb{R}^{M}\right): \frac{\|w(x)-w(y)\|_{\mathbb{R}^{M}}}{\|x-y\|_{\mathbb{R}^{N}}^{\frac{N}{p}+s}} \in L^{p}(\Omega \times \Omega, \mathbb{R})\right\} \\
& =\left\{w \in L^{p}\left(\Omega, \mathbb{R}^{M}\right):|w|_{W^{s, p}\left(\Omega, \mathbb{R}^{M}\right)}<\infty\right\},
\end{aligned}
$$

equipped with the norm

$$
\|\cdot\|_{W^{s, p}\left(\Omega, \mathbb{R}^{M}\right)}:=\left(\|\cdot\|_{L^{p}\left(\Omega, \mathbb{R}^{M}\right)}^{p}+|\cdot|_{W^{s, p}\left(\Omega, \mathbb{R}^{M}\right)}^{p}\right)^{1 / p}
$$

where $|\cdot|_{W^{s, p}\left(\Omega, \mathbb{R}^{M}\right)}$ is the semi-norm for $W^{s, p}\left(\Omega, \mathbb{R}^{M}\right)$, given by

$$
\begin{aligned}
& |w|_{W^{s, p}\left(\Omega, \mathbb{R}^{M}\right)} \\
& :=\left(\int_{\Omega \times \Omega} \frac{\|w(x)-w(y)\|_{\mathbb{R}^{M}}^{p}}{\|x-y\|_{\mathbb{R}^{N}}^{N+p s}} \mathrm{~d}(x, y)\right)^{1 / p} \\
& \quad w \in W^{s, p}\left(\Omega, \mathbb{R}^{M}\right)
\end{aligned}
$$

- For $s=1$ the Sobolev space $W^{1, p}\left(\Omega, \mathbb{R}^{M}\right)$ consists of all weakly differentiable functions in $L^{1}\left(\Omega, \mathbb{R}^{M}\right)$ for which

$$
\begin{aligned}
\|w\|_{W^{1, p}\left(\Omega, \mathbb{R}^{M}\right)}:= & \left(\|w\|_{L^{p}\left(\Omega, \mathbb{R}^{M}\right)}^{p}\right. \\
& \left.+\int_{\Omega}\|\nabla w(x)\|_{\mathbb{R}^{M \times N}}^{p} \mathrm{~d} x\right)^{1 / p}<\infty,
\end{aligned}
$$

where $\nabla w$ is the weak Jacobian of $w$.

- Moreover, we recall one possible definition of the space $B V\left(\Omega, \mathbb{R}^{M}\right)$ from [2], which consists of all LebesgueBorel measurable functions $w: \Omega \rightarrow \mathbb{R}^{M}$ for which

$\|w\|_{B V\left(\Omega, \mathbb{R}^{M}\right)}:=\|w\|_{L^{1}\left(\Omega, \mathbb{R}^{M}\right)}+|w|_{B V\left(\Omega, \mathbb{R}^{M}\right)}<\infty$,

where

$$
\begin{aligned}
& |w|_{B V\left(\Omega, \mathbb{R}^{M}\right)} \\
& :=\sup \left\{\int_{\Omega} w(x) \cdot \operatorname{Div} \varphi(x) \mathrm{d} x: \varphi \in C_{c}^{1}\left(\Omega, \mathbb{R}^{M \times N}\right)\right. \\
& \text { such that } \left.\|\varphi\|_{\infty}:=\underset{x \in \Omega}{\operatorname{ess} \sup }\|\varphi(x)\|_{F} \leq 1\right\}
\end{aligned}
$$

where $\|\varphi(x)\|_{F}$ is the Frobenius-norm of the matrix $\varphi(x)$ and $\operatorname{Div} \varphi=\left(\operatorname{div} \varphi_{1}, \ldots, \operatorname{div} \varphi_{M}\right)^{\mathrm{T}}$ denotes the row-wise formed divergence of $\varphi$.

Lemma 2.4 Let $0<s \leq 1$ and $p \in[1, \infty)$, then $W^{s, p}\left(\Omega, \mathbb{R}^{M}\right) \hookrightarrow L^{p}\left(\Omega, \mathbb{R}^{M}\right)$ and the embedding is compact. Moreover, the embedding $B V\left(\Omega, \mathbb{R}^{M}\right) \hookrightarrow L^{p}\left(\Omega, \mathbb{R}^{M}\right)$ is compact for all

$1 \leq p<1^{*}:=\left\{\begin{array}{ll}+\infty & \text { if } N=1 \\ \frac{N}{N-1} & \text { otherwise }\end{array}\right.$.

Proof The first result can be found in [24] for $0<s<1$ and in [29] for $s=1$. The second assertion is stated in [2].

Remark 2.5 Let Assumption 2.1 hold. We recall some basic properties of weak convergence in $W^{s, p}\left(\Omega, \mathbb{R}^{M}\right)$, $W^{1, p}\left(\Omega, \mathbb{R}^{M}\right)$ and weak* convergence in $B V\left(\Omega, \mathbb{R}^{M}\right)$ (see, for instance, [1,2]):

- Let $p>1, s \in(0,1]$ and assume that $\left(w_{n}\right)_{n \in \mathbb{N}}$ is bounded in $W^{s, p}\left(\Omega, \mathbb{R}^{M}\right)$. Then there exists a subsequence $\left(w_{n_{k}}\right)_{k \in \mathbb{N}}$ which converges weakly in $W^{s, p}\left(\Omega, \mathbb{R}^{M}\right)$.

- Assume that $\left(w_{n}\right)_{n \in \mathbb{N}}$ is bounded in $B V\left(\Omega, \mathbb{R}^{M}\right)$. Then there exists a subsequence $\left(w_{n_{k}}\right)_{k \in \mathbb{N}}$ which converges weakly* in $B V\left(\Omega, \mathbb{R}^{M}\right)$. 
Before introducing the regularization functional, which we investigate theoretically and numerically, we give the definition of some sets of (equivalence classes of) admissible functions.

Definition 2.6 For $0<s \leq 1, p \geq 1$ and a nonempty closed subset $K \subseteq \mathbb{R}^{M}$, we define

$$
\begin{aligned}
L^{p}(\Omega, K) & :=\left\{\phi \in L^{p}\left(\Omega, \mathbb{R}^{M}\right): \phi(x) \in K \text { for a.e. } x \in \Omega\right\} ; \\
W^{s, p}(\Omega, K) & :=\left\{w \in W^{s, p}\left(\Omega, \mathbb{R}^{M}\right): w(x) \in K \text { for a.e. } x \in \Omega\right\}, \\
B V(\Omega, K) & :=\left\{w \in B V\left(\Omega, \mathbb{R}^{M}\right): w(x) \in K \text { for a.e. } x \in \Omega\right\} .
\end{aligned}
$$

and equip each of these (in general nonlinear) sets with some subspace topology:

- $L^{p}(\Omega, K) \subseteq L^{p}\left(\Omega, \mathbb{R}^{M}\right)$ is associated with the strong $L^{p}\left(\Omega, \mathbb{R}^{M}\right)$-topology,

- $W^{s, p}(\Omega, K) \subseteq W^{s, p}\left(\Omega, \mathbb{R}^{M}\right)$ is associated with the weak $W^{s, p}\left(\Omega, \mathbb{R}^{M}\right)$-topology, and

- $B V(\Omega, K) \subseteq B V\left(\Omega, \mathbb{R}^{M}\right)$ is associated with the weak* $B V\left(\Omega, \mathbb{R}^{M}\right)$-topology.

Moreover, we define

$$
W(\Omega, K):= \begin{cases}W^{s, p}(\Omega, K) & \text { for } p \in(1, \infty) \text { and } s \in(0,1], \\ B V(\Omega, K) & \text { for } p=1 \text { and } s=1 .\end{cases}
$$

Consistently, $W(\Omega, K)$

- is associated with the weak $W^{s, p}\left(\Omega, \mathbb{R}^{M}\right)$-topology in the case $p \in(1, \infty)$ and $s \in(0,1]$ and

- with the weak* $B V\left(\Omega, \mathbb{R}^{M}\right)$-topology when $p=1$ and $s=1$.

When we speak about

convergence on $W(\Omega, K)$ we write $\stackrel{W(\Omega, K)}{\longrightarrow}$ or simply $\stackrel{W}{\rightarrow}$ and mean weak convergence on $W^{s, p}(\Omega, K)$ and weak* convergence on $B V(\Omega, K)$, respectively.

Remark 2.7 - In general $L^{p}(\Omega, K), W^{s, p}(\Omega, K)$ and $B V(\Omega, K)$ are sets which do not form a linear space.

- If $K=\mathbb{S}^{1}$, then $W^{s, p}(\Omega, K)=W^{s, p}\left(\Omega, \mathbb{S}^{1}\right)$ as occurred in [13].

- For an embedded manifold $K$, the dimension of the manifold is not necessarily identical with the space dimension of $\mathbb{R}^{M}$. For instance, if $K=\mathbb{S}^{1} \subseteq \mathbb{R}^{2}$, then the dimension of $\mathbb{S}^{1}$ is 1 and $M=2$.

The following lemma shows that $W(\Omega, K)$ is a sequentially closed subset of $W\left(\Omega, \mathbb{R}^{M}\right)$.
Lemma 2.8 (Sequential closedness of $W(\Omega, K)$ and $\left.L^{p}(\Omega, K)\right)$

(i) Let $w_{*} \in W\left(\Omega, \mathbb{R}^{M}\right)$ and $\left(w_{n}\right)_{n \in \mathbb{N}}$ be a sequence in $W(\Omega, K) \subseteq W\left(\Omega, \mathbb{R}^{M}\right)$ with $w_{n} \stackrel{W\left(\Omega, \mathbb{R}^{M}\right)}{\longrightarrow} w_{*}$ as $n \rightarrow$ $\infty$. Then $w_{*} \in W(\Omega, K)$ and $w_{n} \rightarrow w_{*}$ in $L^{p}(\Omega, K)$.

(ii) Let $v_{*} \in L^{p}\left(\Omega, \mathbb{R}^{M}\right)$ and $\left(v_{n}\right)_{n \in \mathbb{N}}$ be a sequence in $L^{p}(\Omega, K) \subseteq L^{p}\left(\Omega, \mathbb{R}^{M}\right)$ with $v_{n} \rightarrow v_{*}$ in $L^{p}\left(\Omega, \mathbb{R}^{M}\right)$ as $n \rightarrow \infty$. Then $v_{*} \in L^{p}(\Omega, K)$ and there is some subsequence $\left(v_{n_{k}}\right)_{k \in \mathbb{N}}$ which converges to $v_{*}$ pointwise almost everywhere, i.e., $v_{n_{k}}(x) \rightarrow v_{*}(x)$ as $k \rightarrow \infty$ for almost every $x \in \Omega$.

Proof For the proof of the second part, cf. [27], Chapter VI, Corollary 2.7, take into account the closedness of $K \subseteq \mathbb{R}^{M}$. The proof of the first part follows from standard convergence arguments in $W^{s, p}\left(\Omega, \mathbb{R}^{M}\right), B V\left(\Omega, \mathbb{R}^{M}\right)$ and $L^{p}\left(\Omega, \mathbb{R}^{M}\right)$, respectively, using the embeddings from Lemma 2.4, an argument on subsequences and part two.

Remark 2.9 Lemma 2.4 along with Lemma 2.8 imply that $W(\Omega, K)$ is compactly embedded in $L^{p}(\Omega, K)$, where these sets are equipped with the bornology inherited from $W\left(\Omega, \mathbb{R}^{M}\right)$ and the topology inherited from $L^{p}\left(\Omega, \mathbb{R}^{M}\right)$, respectively.

In the following we postulate the assumptions on the operator $\mathrm{F}$ which will be used throughout the paper:

Assumption 2.10 Let $W\left(\Omega_{1}, K_{1}\right)$ be as in Eq. 2.5 and assume that $\mathrm{F}$ is an operator from $W\left(\Omega_{1}, K_{1}\right)$ to $L^{p_{2}}\left(\Omega_{2}, K_{2}\right)$.

We continue with the definition of our regularization functionals:

Definition 2.11 Let Assumptions 2.1 and 2.10 hold. Moreover, let $\varepsilon>0$ be fixed and let $\rho:=\rho_{\varepsilon}$ be a mollifier.

The regularization functional $\mathcal{F}_{\alpha}^{v}\left[\mathrm{~d}_{2}, \mathrm{~d}_{1}\right]: W\left(\Omega_{1}, K_{1}\right) \rightarrow$ $[0, \infty]$ is defined as follows

$$
\begin{aligned}
\mathcal{F}_{\alpha}^{v}\left[\mathrm{~d}_{2}, \mathrm{~d}_{1}\right](w):= & \int_{\Omega_{2}} \mathrm{~d}_{2}^{p_{2}}(\mathrm{~F}(w)(x), v(x)) \mathrm{d} x \\
& +\alpha \int_{\Omega_{1} \times \Omega_{1}} \frac{\mathrm{d}_{1}^{p_{1}}(w(x), w(y))}{\|x-y\|_{\mathrm{R}^{N}}^{k+p_{1} s}} \rho^{l}(x-y) \mathrm{d}(x, y),
\end{aligned}
$$

where

(i) $v \in L^{p_{2}}\left(\Omega_{2}, K_{2}\right)$,

(ii) $s \in(0,1]$,

(iii) $\alpha \in(0,+\infty)$ is the regularization parameter,

(iv) $l \in\{0,1\}$ is an indicator and 
(v) $\left\{\begin{aligned} & k \leq N \text { if } W\left(\Omega_{1}, K_{1}\right)=W^{s, p_{1}}\left(\Omega_{1}, K_{1}\right), 0<s<1 \\ & k=0 \text { if } W\left(\Omega_{1}, K_{1}\right)=W^{1, p_{1}}\left(\Omega_{1}, K_{1}\right) \text { or if } \\ & W\left(\Omega_{1}, K_{1}\right)=B V\left(\Omega_{1}, K_{1}\right), \text { respectively. }\end{aligned}\right.$

Setting

$\llbracket \phi, v \rrbracket_{\left[\mathrm{d}_{2}\right]}:=\left(\int_{\Omega_{2}} \mathrm{~d}_{2}^{p_{2}}(\phi(x), v(x)) \mathrm{d} x\right)^{\frac{1}{p_{2}}}$

and

$\mathcal{R}_{\left[\mathrm{d}_{1}\right]}(w):=\int_{\Omega_{1} \times \Omega_{1}} \frac{\mathrm{d}_{1}^{p_{1}}(w(x), w(y))}{\|x-y\|_{\mathbb{R}^{N}}^{k+p_{1} s}} \rho^{l}(x-y) \mathrm{d}(x, y)$,

Equation 2.6 can be expressed in compact form

$\mathcal{F}_{\alpha}^{v}\left[\mathrm{~d}_{2}, \mathrm{~d}_{1}\right](w)=\llbracket \mathrm{F}(w), v \rrbracket_{\left[\mathrm{d}_{2}\right]}^{p_{2}}+\alpha \mathcal{R}_{\left[\mathrm{d}_{1}\right]}(w)$.

For convenience we will often skip some of the super- or subscript and use compact notations like, e.g.,

$\left.\mathcal{F}^{v}, \mathcal{F} \mathrm{d}_{2}, \mathrm{~d}_{1}\right]$ or $\mathcal{F}(w)=\llbracket \mathrm{F}(w), v \rrbracket^{p_{2}}+\alpha \mathcal{R}(w)$.

Remark 2.12 (i) $l=\{0,1\}$ is an indicator which allows to consider approximations of Sobolev semi-norms and double integral representations of the type of Bourgain et al. [14] in a uniform manner.

- when $k=0, s=1, l=1$ and when $d_{1}$ is the Euclidean distance, we get the double integrals of the Bourgain et al.-form [14]. Compare with Eq. 1.3.

- When $d_{1}$ is the Euclidean distance, $k=N$ and $l=0$, we get Sobolev semi-norms.

We expect a relation between the two classes of functionals for $l=0$ and $l=1$ as stated in Sect. 5.2.

(ii) When $d_{1}$ is the Euclidean distance then the second term in Eq. 2.6 is similar to the ones used in $[3,11,14,23,49]$.

In the following we state basic properties of $\llbracket \cdot, \cdot \mathbb{\rrbracket}_{\left[\mathrm{d}_{2}\right]}$ and the functional $\mathcal{F}$.

\section{Proposition 2.13 Let Assumption 2.1 hold.}

(i) Then the mapping $\llbracket \cdot, \cdot \rrbracket_{\left[\mathrm{d}_{2}\right]}: L^{p_{2}}\left(\Omega_{2}, K_{2}\right) \times L^{p_{2}}\left(\Omega_{2}, K_{2}\right)$ $\rightarrow[0,+\infty]$ satisfies the metric axioms.

(ii) Let, in addition, Assumption 2.10 hold, assume that $v \in L^{p_{2}}\left(\Omega_{2}, K_{2}\right)$ and that both metrics $d_{i}, i=1,2$, are equivalent to $\left.\mathrm{d}_{\mathbb{R}^{M_{i}}}\right|_{K_{i} \times K_{i}}$, respectively. Then the functional $\mathcal{F}_{\alpha}^{v}\left[\mathrm{~d}_{2}, \mathrm{~d}_{1}\right]$ does not attain the value $+\infty$ on its domain $W\left(\Omega_{1}, K_{1}\right) \neq \emptyset$.
Proof (i) The axioms of non-negativity, identity of indiscernibles and symmetry are fulfilled by $\llbracket \cdot, \cdot \rrbracket_{\left[\mathrm{d}_{2}\right]}$ since $\mathrm{d}_{2}$ is a metric. To prove the triangle inequality, let $\phi, \xi, v \in$ $L^{p_{2}}\left(\Omega_{2}, K_{2}\right)$. In the main case $\llbracket \phi, v \rrbracket_{\left[\mathrm{d}_{2}\right]}^{p_{2}} \in(0, \infty)$ Hölder's inequality yields

$$
\begin{aligned}
& \llbracket \phi, v \rrbracket \\
&= \int_{\Omega_{2}}^{p_{2}} \mathrm{~d}_{2}(\phi(x), v(x)) \mathrm{d}_{2}^{p_{2}-1}(\phi(x), v(x)) \mathrm{d} x \\
& \leq \int_{\Omega_{2}} \mathrm{~d}_{2}(\phi(x), \xi(x)) \mathrm{d}_{2}^{p_{2}-1}(\phi(x), v(x)) \mathrm{d} x \\
&+\int_{\Omega_{2}} \mathrm{~d}_{2}(\xi(x), v(x)) \mathrm{d}_{2}^{p_{2}-1}(\phi(x), v(x)) \mathrm{d} x \\
& \leq\left(\int_{\Omega_{2}} \mathrm{~d}_{2}^{p_{2}}(\phi(x), \xi(x)) \mathrm{d} x\right)^{\frac{1}{p_{2}}}\left(\int_{\Omega_{2}} \mathrm{~d}_{2}^{p_{2}}(\phi(x), v(x)) \mathrm{d} x\right)^{\frac{p_{2}-1}{p_{2}}} \\
&+\left(\int_{\Omega_{2}} \mathrm{~d}_{2}^{p_{2}}(\xi(x), v(x)) \mathrm{d} x\right)^{\frac{1}{p_{2}}}\left(\int_{\Omega_{2}} \mathrm{~d}_{2}^{p_{2}}(\phi(x), v(x)) \mathrm{d} x\right)^{\frac{p_{2}-1}{p_{2}}} \\
&=\left(\llbracket \phi, \xi \rrbracket_{\left[\mathrm{d}_{2}\right]}+\llbracket \xi, v \rrbracket\left[\mathrm{d}_{2}\right]\right) \llbracket \phi, v \rrbracket_{\left[\mathrm{d}_{2}\right]}^{p_{2}-1},
\end{aligned}
$$

meaning

$\llbracket \phi, v \rrbracket_{\left[\mathrm{d}_{2}\right]} \leq \llbracket \phi, \xi \rrbracket_{\left[\mathrm{d}_{2}\right]}+\llbracket \xi, v \rrbracket_{\left[\mathrm{d}_{2}\right]}$.

If $\llbracket \phi, v \rrbracket_{\left[\mathrm{d}_{2}\right]}=0$, the triangle inequality is trivially fulfilled.

In the remaining case $\llbracket \phi, v \rrbracket_{\left[\mathrm{d}_{2}\right]}=\infty$ applying the estimate $(a+b)^{p} \leq 2^{p-1}\left(a^{p}+b^{p}\right)$, see, e.g., [55, Lemma 3.20], to $a=\mathrm{d}_{2}(\phi(x), \xi(x)) \geq 0$ and $b=$ $\mathrm{d}_{2}(\xi(x), v(x)) \geq 0$ yields

$\llbracket \phi, v \rrbracket_{\left[\mathrm{d}_{2}\right]}^{p_{2}} \leq 2^{p_{2}-1}\left(\llbracket \phi, \xi \rrbracket_{\left[\mathrm{d}_{2}\right]}^{p_{2}}+\llbracket \xi, v \rrbracket_{\left[\mathrm{d}_{2}\right]}^{p_{2}}\right)$,

implying the desired result.

(ii) We emphasize that $W\left(\Omega_{1}, K_{1}\right) \neq \varnothing$ because every constant function $w(\cdot)=a \in K_{1}$ belongs to $W^{s, p_{1}}\left(\Omega_{1}, K_{1}\right)$ for $p_{1} \in(1, \infty)$ and $s \in(0,1]$ as well as to $B V\left(\Omega_{1}, K_{1}\right)$ for $p_{1}=1$ and $s=1$. Assume now that the metrics $d_{i}$ are equivalent to $\left.\mathrm{d}_{\mathbb{R}^{M_{i}}}\right|_{K_{i} \times K_{i}}$ for $i=1$ and $i=2$, respectively, so that we have an upper bound $d_{i} \leq\left. C \mathrm{~d}_{\mathbb{R}^{M_{i}}}\right|_{K_{i} \times K_{i}}$. We need to prove that $\mathcal{F}_{\alpha}^{v}\left[\mathrm{~d}_{2}, \mathrm{~d}_{1}\right](w)<\infty$ for every $w \in W\left(\Omega_{1}, K_{1}\right)$. Due to $\llbracket \phi, v \rrbracket_{\left[\mathrm{d}_{2}\right]}^{p_{2}} \leq C^{p_{2}}\|\phi-v\|_{L^{p_{2}\left(\Omega_{2}, \mathbb{R}^{\left.M_{2}\right)}\right.}}^{p_{2}}<\infty$ for all $\phi, v \in L^{p_{2}}\left(\Omega_{2}, K_{2}\right) \subseteq L^{p_{2}}\left(\Omega_{2}, \mathbb{R}^{M_{2}}\right)$ it is sufficient to show $\mathcal{R}_{\left[\mathrm{d}_{1}\right]}(w)<+\infty$ for all $w \in W\left(\Omega_{1}, K_{1}\right)$.

- For $W\left(\Omega_{1}, K_{1}\right)=B V\left(\Omega_{1}, K_{1}\right)$ this is guaranteed by [49, Theorem 1.2].

- For $W\left(\Omega_{1}, K_{1}\right)=W^{1, p_{1}}\left(\Omega_{1}, K_{1}\right)$ by $[14$, Theorem $1]$. 
- For $W\left(\Omega_{1}, K_{1}\right)=W^{s, p_{1}}\left(\Omega_{1}, K_{1}\right), s \in(0,1)$, we distinguish between two cases.

If $\|x-y\|_{\mathbb{R}^{N}}<1$, we have that $\frac{1}{\|x-y\|_{\mathbb{R}^{N}}^{k+p_{1} s}} \leq$ $\frac{1}{\|x-y\|_{\mathbb{R}^{N}}^{N+p_{1} s}}$ for $k \leq N$ and hence

$$
\begin{aligned}
& \int_{\substack{(x, y) \in \Omega_{1} \times \Omega_{1} \\
\|x-y\|_{\mathbb{R}^{N}<1}}} \frac{\mathrm{d}_{1}^{p_{1}}(w(x), w(y))}{\|x-y\|_{\mathbb{R}^{N}}^{k+p_{1} s}} \rho^{l}(x-y) \mathrm{d}(x, y) \\
& \leq C^{p_{1}}\left\|\rho^{l}\right\|_{\infty}|w|_{W^{s, p_{1}}\left(\Omega_{1}, \mathbb{R}^{\left.M_{1}\right)}\right.}^{p_{1}}<\infty .
\end{aligned}
$$

If $\|x-y\|_{\mathbb{R}^{N}} \geq 1$, we can estimate

$$
\begin{aligned}
& \int_{\substack{(x, y) \in \Omega_{1} \times \Omega_{1} \\
\|x-y\|_{\mathbb{R}^{N} \geq 1}}} \frac{\mathrm{d}_{1}^{p_{1}}(w(x), w(y))}{\|x-y\|_{\mathbb{R}^{N}}^{k+p_{1} s}} \rho^{l}(x-y) \mathrm{d}(x, y) \\
& \leq C^{p_{1}}\left\|\rho^{l}\right\|_{\infty} 2^{p_{1}}\left|\Omega_{1}\right|\|w\|_{L^{p_{1}}\left(\Omega_{1}, \mathbb{R}^{M_{1}}\right)}^{p_{1}}<\infty .
\end{aligned}
$$

In summary adding yields $\mathcal{R}_{\left[\mathrm{d}_{1}\right]}(w)<+\infty$.

\section{Existence}

In order to prove existence of a minimizer of the functional $\mathcal{F}$, we apply the direct method in the Calculus of Variations (see, e.g., [20,21]). To this end we verify continuity properties of $\llbracket \cdot, \cdot \rrbracket_{\left[\mathrm{d}_{2}\right]}$ and $\mathcal{R}_{\left[\mathrm{d}_{1}\right]}$, resp. $\mathcal{F}\left[\mathrm{d}_{2}, \mathrm{~d}_{1}\right]$ and apply them along with the sequential closedness of $W\left(\Omega_{1}, K_{1}\right)$, already proven in Lemma 2.8.

In this context we point out some setting assumptions and their consequences on $\mathcal{F}$, resp. $\llbracket \cdot, \cdot \rrbracket$ and $\mathcal{R}$ in the following remark. For simplicity we assume $p:=p_{1}=p_{2} \in(1, \infty)$, $\Omega:=\Omega_{1}=\Omega_{2}$ and $\left(K, \mathrm{~d}_{K}\right):=\left(K_{1}, \mathrm{~d}_{1}\right)=\left(K_{2}, \mathrm{~d}_{2}\right)$.

Remark 3.1 - The continuity of $\mathrm{d}_{K}$ with respect to $\left.\mathrm{d}_{\mathbb{R}^{M}}\right|_{K \times K}$ guarantees lower semicontinuity of $\llbracket \cdot, \cdot \rrbracket\left[\mathrm{d}_{K}\right]$ and $\mathcal{R}_{\left[\mathrm{d}_{K}\right]}$.

- The inequality $\left.\mathrm{d}_{\mathbb{R}^{M}}\right|_{K \times K} \leq \mathrm{d}_{K}$ carries over to the inequalities $\|\widetilde{v}-v\|_{L^{p}\left(\Omega, \mathbb{R}^{M}\right)} \leq \llbracket \widetilde{v}, v \rrbracket_{\left[\mathrm{d}_{K}\right]}$ for all $\tilde{v}, v \in$ $L^{p}(\Omega, K)$, and $|w|_{W\left(\Omega, \mathbb{R}^{M}\right)} \leq \mathcal{R}_{\left[\mathrm{d}_{K}\right]}(w)$ for all $w \in$ $W(\Omega, K)$, allowing to transfer properties like coercivity from $\mathcal{F}\left[\mathrm{d}_{\mathbb{R}^{M}}, \mathrm{~d}_{\mathbb{R}^{M}}\right]$ to $\left.\mathcal{F} \mathrm{d}_{K}, \mathrm{~d}_{K}\right]$. Moreover, the extended real-valued metric space $\left(L^{p}(\Omega, K), \llbracket \cdot \cdot \cdot \rrbracket_{\left[\mathrm{d}_{K}\right]}\right)$ stays related to the linear space $\left(L^{p}\left(\Omega, \mathbb{R}^{M}\right),\|\cdot\|_{L^{p}\left(\Omega, \mathbb{R}^{M}\right)}\right)$ in terms of the topology and bornology induced by $\llbracket \cdot, \cdot \rrbracket$, resp. those inherited by $\|\cdot\|_{L^{p}\left(\Omega, \mathbb{R}^{M}\right)}$.

- The closedness of $K \subseteq \mathbb{R}^{M}$ is crucial in showing that $W(\Omega, K)$ is a sequentially closed subset of the linear space $W\left(\Omega, \mathbb{R}^{M}\right)$. This closedness property acts as a kind of replacement for the, a priori not available, notion of completeness with respect to the "space" $(W(\Omega, K), \llbracket \cdot, \cdot \rrbracket, \mathcal{R})$.

For $l=0, k=N$ note in the latter item that equipping $W(\Omega, K)$ with $\llbracket \cdot, \cdot \rrbracket_{\left[\mathrm{d}_{2}\right]}$ and $\mathcal{R}_{\left[\mathrm{d}_{1}\right]}$ does not even lead to an (extended real-valued) metric space, in contrast to the classical case $\left(K, \mathrm{~d}_{K}\right)=\left(\mathbb{R}^{M}, \mathrm{~d}_{\mathbb{R}^{M}}\right)$.

We will use the following assumption:

Assumption 3.2 Let Assumption 2.1 hold, $v^{0} \in L^{p_{2}}\left(\Omega_{2}, K_{2}\right)$ and let $W\left(\Omega_{1}, K_{1}\right)$ and the associated topology be as defined in Eq. 2.5.

In addition we assume:

- F:W( $\left.\Omega_{1}, K_{1}\right) \rightarrow L^{p_{2}}\left(\Omega_{2}, K_{2}\right)$ is well defined and sequentially continuous with respect to the specified topology on $W\left(\Omega_{1}, K_{1}\right)$ and

- For every $t>0$ and $\alpha>0$, the level sets

$$
\operatorname{level}_{t}\left(\mathcal{F}_{\alpha}^{v^{0}}\left[\mathrm{~d}_{2}, \mathrm{~d}_{1}\right]\right):=\left\{w \in W\left(\Omega_{1}, K_{1}\right): \mathcal{F}_{\alpha}^{v^{0}}\left[\mathrm{~d}_{2}, \mathrm{~d}_{1}\right] \leq t\right\}
$$

are sequentially pre-compact subsets of $W\left(\Omega_{1}, \mathbb{R}^{M_{1}}\right)$.

- There exists a $\bar{t}>0$ such that $\operatorname{level}_{\bar{t}}\left(\mathcal{F}_{\alpha}^{v^{0}}\left[\mathrm{~d}_{2}, \mathrm{~d}_{1}\right]\right)$ is nonempty.

- Only those $v \in L^{p_{2}}\left(\Omega_{2}, K_{2}\right)$ are considered which additionally fulfill $\llbracket v, v^{0} \rrbracket_{\left[\mathrm{d}_{2}\right]}<\infty$.

Remark 3.3 The third condition is sufficient to guarantee $\mathcal{F}_{\alpha}^{v^{0}}\left[\mathrm{~d}_{2}, \mathrm{~d}_{1}\right] \not \equiv \infty$. In contrast, the condition $v^{0} \in$ $L^{p_{2}}\left(\Omega_{2}, K_{2}\right)$, cf. Definition 2.11, might not be sufficient if $d_{2}$ is not equivalent to $\left.\mathrm{d}_{\mathbb{R}^{M_{2}}}\right|_{K_{2} \times K_{2}}$.

Lemma 3.4 Let Assumption 3.2 hold. Then the mappings $\llbracket \cdot, \cdot \rrbracket_{\left[\mathrm{d}_{2}\right]}, \mathcal{R}_{\left[\mathrm{d}_{1}\right]}$ and $\left.\mathcal{F} \mathrm{d}_{2}, \mathrm{~d}_{1}\right]$ have the following continuity properties:

(i) The mapping $\llbracket \cdot \cdot \cdot \rrbracket_{\left[\mathrm{d}_{2}\right]}: L^{p_{2}}\left(\Omega_{2}, K_{2}\right) \times L^{p_{2}}\left(\Omega_{2}, K_{2}\right) \rightarrow$ $[0,+\infty]$ is sequentially lower semi-continuous, i.e., whenever sequences $\left(\phi_{n}\right)_{n \in \mathbb{N}},\left(v_{n}\right)_{n \in \mathbb{N}}$ in $L^{p_{2}}\left(\Omega_{2}, K_{2}\right)$ converge to $\phi_{*} \in L^{p_{2}}\left(\Omega_{2}, K_{2}\right)$ and $\nu_{*} \in L^{p_{2}}\left(\Omega_{2}, K_{2}\right)$, respectively, we have $\llbracket \phi_{*}, v_{*} \rrbracket_{\left[\mathrm{d}_{2}\right]} \leq \liminf _{n \rightarrow \infty} \llbracket \phi_{n}, v_{n} \rrbracket_{\left[\mathrm{d}_{2}\right]}$.

(ii) The functional $\mathcal{R}_{\left[\mathrm{d}_{1}\right]}: W\left(\Omega_{1}, K_{1}\right) \rightarrow[0, \infty]$ is sequentially lower semi-continuous, i.e., whenever a sequence $\left(w_{n}\right)_{n \in \mathbb{N}}$ in $W\left(\Omega_{1}, K_{1}\right)$ converges to some $w_{*} \in W\left(\Omega_{1}, K_{1}\right)$ we have

$\mathcal{R}_{\left[\mathrm{d}_{1}\right]}\left(w_{*}\right) \leq \liminf _{n \rightarrow \infty} \mathcal{R}_{\left[\mathrm{d}_{1}\right]}\left(w_{n}\right)$.

(iii) The functional $\mathcal{F}\left[\mathrm{d}_{2}, \mathrm{~d}_{1}\right]: W\left(\Omega_{1}, K_{1}\right) \rightarrow[0, \infty]$ is sequentially lower semi-continuous. 
Proof (i) It is sufficient to show that for every pair of sequences $\left(\phi_{n}\right)_{n \in \mathbb{N}},\left(v_{n}\right)_{n \in \mathbb{N}}$ in $L^{p_{2}}\left(\Omega_{2}, K_{2}\right)$ which converge to previously fixed elements $\phi_{*} \in L^{p_{2}}\left(\Omega_{2}, K_{2}\right)$ and $v_{*} \in L^{p_{2}}\left(\Omega_{2}, K_{2}\right)$, respectively, we can extract subsequences $\left(\phi_{n_{j}}\right)_{j \in \mathbb{N}}$ and $\left(v_{n_{j}}\right)_{j \in \mathbb{N}}$, respectively, with

$\llbracket \phi_{*}, v_{*} \rrbracket_{\left[\mathrm{d}_{2}\right]} \leq \liminf _{j \rightarrow \infty} \llbracket \phi_{n_{j}}, v_{n_{j}} \rrbracket_{\left[\mathrm{d}_{2}\right]}$.

To this end let $\left(\phi_{n}\right)_{n \in \mathbb{N}},\left(v_{n}\right)_{n \in \mathbb{N}}$ be some sequences in $L^{p_{2}}\left(\Omega_{2}, K_{2}\right)$ with $\phi_{n} \rightarrow \phi_{*}$ and $v_{n} \rightarrow v_{*}$ in $L^{p_{2}}\left(\Omega_{2}, K_{2}\right)$. Lemma 2.8 ensures that there exist subsequences $\left(\phi_{n_{j}}\right)_{j \in \mathbb{N}},\left(v_{n_{j}}\right)_{j \in \mathbb{N}}$ converging to $\phi_{*}$ and $\nu_{*}$ pointwise almost everywhere, which in turn implies $\left(\phi_{n_{j}}(\cdot), v_{n_{j}}(\cdot)\right) \rightarrow\left(\phi_{*}(\cdot), v_{*}(\cdot)\right)$ pointwise almost everywhere. Therefrom, together with the continuity of $\mathrm{d}_{2}$ : $K_{2} \times K_{2} \rightarrow[0, \infty)$ with respect to $\mathrm{d}_{\mathbb{R}^{M_{2}}}$, cf. Sect. 2, we obtain by using the quadrangle inequality that

$$
\begin{aligned}
& \left|\mathrm{d}_{2}\left(\phi_{n_{j}}(x), v_{n_{j}}(x)\right)-\mathrm{d}_{2}\left(\phi_{*}(x), v_{*}(x)\right)\right| \\
& \quad \leq \mathrm{d}_{2}\left(\phi_{n_{j}}(x), \phi_{*}(x)\right)+\mathrm{d}_{2}\left(v_{n_{j}}(x), v_{*}(x)\right) \rightarrow 0,
\end{aligned}
$$

and hence

$$
\mathrm{d}_{2}^{p_{2}}\left(\phi_{n_{j}}(x), v_{n_{j}}(x)\right) \rightarrow \mathrm{d}_{2}^{p_{2}}\left(\phi_{*}(x), v_{*}(x)\right)
$$

for almost every $x \in \Omega_{2}$.

Applying Fatou's lemma, we obtain

$$
\begin{aligned}
\llbracket \phi_{*}, v_{*} \rrbracket_{\left[\mathrm{d}_{2}\right]} & =\int_{\Omega_{2}} \mathrm{~d}_{2}^{p_{2}}\left(\phi_{*}(x), v_{*}(x)\right) \mathrm{d} x \\
& \leq \liminf _{j \rightarrow \infty} \int_{\Omega_{2}} \mathrm{~d}_{2}^{p_{2}}\left(\phi_{n_{j}}(x), v_{n_{j}}(x)\right) \mathrm{d} x \\
& =\liminf _{j \rightarrow \infty} \llbracket \phi_{n_{j}}, v_{n_{j}} \rrbracket_{\left[\mathrm{d}_{2}\right]} .
\end{aligned}
$$

(ii) Let $\left(w_{n}\right)_{n \in \mathbb{N}}$ be a sequence in $W\left(\Omega_{1}, K_{1}\right)$ with $w_{n} \stackrel{W}{\rightarrow}$ $w_{*}$ as $n \rightarrow \infty$. By Lemma 2.8 there is a subsequence $\left(w_{n_{j}}\right)_{j \in \mathbb{N}}$ which converges to $w_{*}$ both in $L^{p_{1}}\left(\Omega_{1}, K_{1}\right)$ and pointwise almost everywhere. This further implies that

$\mathrm{d}_{1}^{p_{1}}\left(w_{n_{j}}(x), w_{n_{j}}(y)\right) \rightarrow \mathrm{d}_{1}^{p_{1}}\left(w_{*}(x), w_{*}(y)\right)$

for almost every

$(x, y) \in \Omega_{1} \times \Omega_{1} \supseteq\left\{(x, y) \in \Omega_{1} \times \Omega_{1}: x \neq y\right\}=: A$.

$$
\begin{aligned}
& f_{j}(x, y) \\
& := \begin{cases}\frac{\mathrm{d}_{1}^{p_{1}}\left(w_{n_{j}}(x), w_{n_{j}}(y)\right)}{\|x-y\|_{\mathbb{R}^{N}}^{k+p s}} \rho^{l}(x-y) & \text { for }(x, y) \in A, \\
0 & \text { for }(x, y) \in\left(\Omega_{1} \times \Omega_{1}\right) \backslash A,\end{cases}
\end{aligned}
$$

for all $j \in \mathbb{N}$ and

$$
\begin{aligned}
& f_{*}(x, y) \\
& := \begin{cases}\frac{\mathrm{d}_{1}^{p_{1}}\left(w_{*}(x), w_{*}(y)\right)}{\|x-y\|_{\mathrm{R}^{N}}^{k+p s}} \rho^{l}(x-y) & \text { for }(x, y) \in A, \\
0 & \text { for }(x, y) \in\left(\Omega_{1} \times \Omega_{1}\right) \backslash A,\end{cases}
\end{aligned}
$$

we thus have $f_{*}(x, y)=\lim _{j \rightarrow \infty} f_{j}(x, y)$ for almost every $(x, y) \in \Omega_{1} \times \Omega_{1}$. Applying Fatou's lemma to the functions $f_{j}$ yields the assertion, due to the same reduction as in the proof of the first part.

(iii) It is sufficient to prove that the components $\mathcal{G}(\cdot)=$ $\llbracket F(\cdot), v \rrbracket_{\left[\mathrm{d}_{2}\right]}$ and $\mathcal{R}=\mathcal{R}_{\left[\mathrm{d}_{1}\right]}$ of $\mathcal{F}\left[\mathrm{d}_{1}, \mathrm{~d}_{2}\right]=\mathcal{G}+\alpha \mathcal{R}$ are sequentially lower semi-continuous. To prove that $\mathcal{G}$ is sequentially lower semi-continuous in every $w_{*} \in$ $W\left(\Omega_{1}, K_{1}\right)$, let $\left(w_{n}\right)_{n \in \mathbb{N}}$ be a sequence in $W\left(\Omega_{1}, K_{1}\right)$ with $w_{n} \stackrel{W}{\rightarrow} w_{*}$ as $n \rightarrow \infty$. Assumption 3.2, ensuring the sequential continuity of $\mathrm{F}: W\left(\Omega_{1}, K_{1}\right) \rightarrow$ $L^{p_{2}}\left(\Omega_{2}, K_{2}\right)$, implies hence $\mathrm{F}\left(w_{n}\right) \rightarrow \mathrm{F}\left(w_{*}\right)$ in $L^{p_{2}}\left(\Omega_{2}, K_{2}\right)$ as $n \rightarrow \infty$. By item $(i)$ we thus obtain $\mathcal{G}\left(w_{*}\right)=\llbracket \mathrm{F}\left(w_{*}\right), v \rrbracket \leq \liminf _{n \rightarrow \infty} \llbracket \mathrm{F}\left(w_{n}\right), v \rrbracket=$ $\liminf _{n \rightarrow \infty} \mathcal{G}\left(w_{n}\right)$.

$\mathcal{R}$ is sequentially lower semi-continuous by item (ii).

\section{Existence of Minimizers}

The proof of the existence of a minimizer of $\mathcal{F}\left[\mathrm{d}_{2}, \mathrm{~d}_{1}\right]$ is along the lines of the proof in [55], taking into account Remark 3.1. We will need the following useful lemma, cf. [55], which links level ${ }_{t}\left(\mathcal{F}_{\alpha}^{v^{0}}\right)$ and $\operatorname{level}_{t}\left(\mathcal{F}_{\alpha}^{v}\right)$ for $\llbracket v, v^{0} \rrbracket<\infty$.

\section{Lemma 3.5 It holds}

$\mathcal{F}^{v_{\star}}\left[\mathrm{d}_{2}, \mathrm{~d}_{1}\right](w) \leq 2^{p_{2}-1} \mathcal{F}^{v_{\diamond}}\left[\mathrm{d}_{2}, \mathrm{~d}_{1}\right](w)+2^{p_{2}-1} \llbracket v_{\diamond}, v_{\star} \rrbracket_{\left[\mathrm{d}_{2}\right]}^{p_{2}}$

for every $w \in W\left(\Omega_{1}, K_{1}\right)$ and $v_{\star}, v_{\diamond} \in L^{p_{2}}\left(\Omega_{2}, K_{2}\right)$.

Proof Using the fact that for $p \geq 1$ we have that $|a+b|^{p} \leq$ $2^{p-1}\left(|a|^{p}+|b|^{p}\right), a, b \in \mathbb{R} \cup\{\infty\}$ and that $\llbracket \cdot, \cdot \rrbracket_{\left[\mathrm{d}_{2}\right]}$ fulfills the triangle inequality, we obtain

$$
\begin{aligned}
\mathcal{F}^{v_{\star}}\left[\mathrm{d}_{2}, \mathrm{~d}_{1}\right](w)= & \llbracket \mathrm{F}(w), v_{\star} \rrbracket_{\left[\mathrm{d}_{2}\right]}^{p_{2}}+\alpha \mathcal{R}_{\left[\mathrm{d}_{1}\right]}(w) \\
\leq & 2^{p_{2}-1}\left(\llbracket \mathrm{F}(w), v_{\diamond} \rrbracket_{\left[\mathrm{d}_{2}\right]}^{p_{2}}+\llbracket v_{\diamond}, v_{\star} \rrbracket_{\left[\mathrm{d}_{2}\right]}^{p_{2}}\right) \\
& +\alpha \mathcal{R}_{\left[\mathrm{d}_{1}\right]}(w) \\
\leq & 2^{p_{2}-1}\left(\mathcal{F}^{v_{\diamond}}\left[\mathrm{d}_{2}, \mathrm{~d}_{1}\right](w)+\llbracket v_{\diamond}, v_{\star} \rrbracket_{\left[\mathrm{d}_{2}\right]}^{p_{2}}\right) .
\end{aligned}
$$

Defining 
Theorem 3.6 Let Assumption 3.2 hold. Then the functional $\mathcal{F}_{\alpha}^{v}\left[\mathrm{~d}_{2}, \mathrm{~d}_{1}\right]: W\left(\Omega_{1}, K_{1}\right) \rightarrow[0, \infty]$ attains a minimizer.

Proof We prove the existence of a minimizer via the direct method. We shortly write $\mathcal{F}^{v}$ for $\mathcal{F}_{\alpha}^{v}\left[\mathrm{~d}_{2}, \mathrm{~d}_{1}\right]$. Let $\left(w_{n}\right)_{n \in \mathbb{N}}$ be a sequence in $W\left(\Omega_{1}, K_{1}\right)$ with

$\lim _{n \rightarrow \infty} \mathcal{F}^{v}\left(w_{n}\right)=\inf _{w \in W\left(\Omega_{1}, K_{1}\right)} \mathcal{F}^{v}(w)$

The latter infimum is not $+\infty$, because $\mathcal{F}^{v} \equiv+\infty$ would imply also $\mathcal{F}^{v^{0}} \equiv+\infty$ due to Lemma 3.5 , violating Assumption 3.2. In particular, there is some $c \in$ $\mathbb{R}$ such that $\mathcal{F}^{v}\left(w_{n}\right) \leq c$ for every $n \in \mathbb{N}$. Applying Lemma 3.5 yields $\mathcal{F}^{v^{0}}\left(w_{n}\right) \leq 2^{p_{2}-1}\left(\mathcal{F}^{v}\left(w_{n}\right)+\llbracket v, v^{0} \rrbracket\right) \leq$ $2^{p_{2}-1}\left(c+\llbracket v, v^{0} \rrbracket\right)=: \tilde{c}<\infty$ due to Assumption 3.2. Since the level set $\operatorname{level}_{\tilde{c}}\left(\mathcal{F}^{v^{0}}\right)$ is sequentially pre-compact with respect to the topology given to $W\left(\Omega_{1}, \mathbb{R}^{M_{1}}\right)$ we get the existence of a subsequence $\left(w_{n_{k}}\right)_{k \in \mathbb{N}}$ which converges to some $w_{*} \in W\left(\Omega_{1}, \mathbb{R}^{M_{1}}\right)$, where actually $w_{*} \in$ $W\left(\Omega_{1}, K_{1}\right)$ due to Lemma 2.8 . Because $\mathcal{F}^{v}$ is sequentially lower semi-continuous, see Lemma 3.4, we have $\mathcal{F}^{v}\left(w_{*}\right) \leq$ $\lim _{\inf _{k \rightarrow \infty}} \mathcal{F}^{v}\left(w_{n_{k}}\right)$. Combining this with Eq. 3.3 we obtain

$$
\begin{aligned}
& \inf _{w \in W\left(\Omega_{1}, K_{1}\right)} \mathcal{F}^{v}(w) \leq \mathcal{F}^{v}\left(w_{*}\right) \\
& \leq \liminf _{k \rightarrow \infty} \mathcal{F}^{v}\left(w_{n_{k}}\right)=\lim _{n \rightarrow \infty} \mathcal{F}^{v}\left(w_{n}\right)=\inf _{w \in W\left(\Omega_{1}, K_{1}\right)} \mathcal{F}^{v}(w) .
\end{aligned}
$$

In particular, $\mathcal{F}^{v}\left(w_{*}\right)=\inf _{w \in W\left(\Omega_{1}, K_{1}\right)} \mathcal{F}^{v}(w)$, meaning that $w_{*}$ is a minimizer of $\mathcal{F}^{v}$.

In the following we investigate two examples, which are relevant for the numerical examples in Sect. 6.

Example 3.7 We consider that $W\left(\Omega_{1}, K_{1}\right)=W^{s, p_{1}}\left(\Omega_{1}, K_{1}\right)$ with $p_{1}>1,0<s<1$ and fix $k=N$.

If the operator $\mathrm{F}$ is norm coercive in the sense that the implication

$$
\left\|w_{n}\right\|_{L^{p_{1}}\left(\Omega_{1}, \mathbb{R}^{\left.M_{1}\right)}\right.} \rightarrow+\infty \Rightarrow\left\|\mathrm{F}\left(w_{n}\right)\right\|_{L^{p_{2}\left(\Omega_{2}, \mathbb{R}^{\left.M_{2}\right)}\right.}} \rightarrow+\infty
$$

holds true for every sequence $\left(w_{n}\right)_{n \in \mathbb{N}}$ in $W^{s, p_{1}}\left(\Omega_{1}, K_{1}\right) \subseteq$ $W^{s, p_{1}}\left(\Omega_{1}, \mathbb{R}^{M_{1}}\right)$, then the functional $\mathcal{F}\left[\mathrm{d}_{2}, \mathrm{~d}_{1}\right]$ : $W^{s, p_{1}}\left(\Omega_{1}, K_{1}\right) \rightarrow[0, \infty]:$

$\mathcal{F}\left[\mathrm{d}_{2}, \mathrm{~d}_{1}\right]=\llbracket \mathrm{F}(w), v \rrbracket_{\left[\mathrm{d}_{2}\right]}^{p_{2}}+\alpha \mathcal{R}_{\left[\mathrm{d}_{1}\right]}(w)$

is coercive. This can be seen as follows:

The inequality between $\mathrm{d}_{1}$ and $\left.\mathrm{d}_{\mathbb{R}^{M_{1}}}\right|_{K_{1} \times K_{1}}$ resp. $\mathrm{d}_{2}$ and $\left.\mathrm{d}_{\mathbb{R}^{M_{2}}}\right|_{K_{2} \times K_{2}}$, see Assumption 2.1, carries over to $\mathcal{F}\left[\mathrm{d}_{2}, \mathrm{~d}_{1}\right]$ and $\left.\mathcal{F} \mathrm{d}_{\mathbb{R}^{M_{2}}}\right|_{K_{2} \times K_{2}},\left.\mathrm{~d}_{\mathbb{R}^{M_{1}}}\right|_{K_{1} \times K_{1}}$, i.e.,

$\mathcal{F}\left[\mathrm{d}_{2}, \mathrm{~d}_{1}\right](w) \geq \mathcal{F}\left[\left.\mathrm{d}_{\mathbb{R}^{M_{2}}}\right|_{K_{2} \times K_{2}},\left.\mathrm{~d}_{\mathbb{R}^{M_{1}}}\right|_{K_{1} \times K_{1}}\right](w)$ for all $w \in W^{s, p_{1}}\left(\Omega_{1}, K_{1}\right)$.

Thus, it is sufficient to show that $\left.\mathcal{F} \mathrm{d}_{\mathbb{R}^{M_{2}}}\right|_{K_{2} \times K_{2}}$, $\left.\left.\mathrm{d}_{\mathbb{R}^{M_{1}}}\right|_{K_{1} \times K_{1}}\right]: W^{s, p_{1}}\left(\Omega_{1}, K_{1}\right) \rightarrow[0, \infty]$ is coercive: To prove this, we write shortly $\mathcal{F}$ instead of $\mathcal{F}\left[\left.\mathrm{d}_{\mathbb{R}^{M_{2}}}\right|_{K_{2} \times K_{2}}\right.$, $\left.\left.\mathrm{d}_{\mathbb{R}^{M_{1}}}\right|_{K_{1} \times K_{1}}\right]$ and consider sequences $\left(w_{n}\right)_{n \in \mathbb{N}}$ in $W^{s, p_{1}}\left(\Omega_{1}, K_{1}\right)$ with $\left\|w_{n}\right\|_{W^{s, p_{1}}\left(\Omega_{1}, \mathbb{R}^{\left.M_{1}\right)}\right.} \rightarrow+\infty$ as $n \rightarrow \infty$. We show that $\mathcal{F}\left(w_{n}\right) \rightarrow+\infty$, as $n \rightarrow \infty$. Since

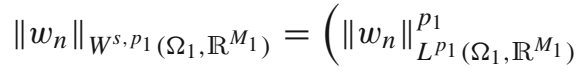

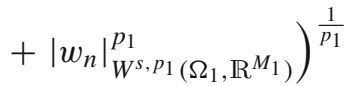

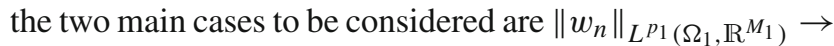

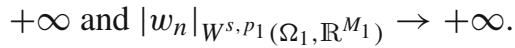

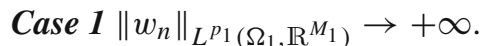

The inverse triangle inequality and the norm coercivity

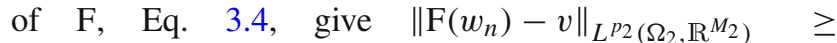
$\left\|\mathrm{F}\left(w_{n}\right)\right\|_{L^{p_{2}\left(\Omega_{2}, \mathbb{R}^{M_{2}}\right)}}-\|v\|_{L^{p_{2}\left(\Omega_{2}, \mathbb{R}^{\left.M_{2}\right)}\right.}} \rightarrow+\infty$. Therefore, also

$$
\begin{aligned}
\mathcal{F}\left(w_{n}\right)= & \left\|\mathrm{F}\left(w_{n}\right)-v\right\|_{L^{p_{2}\left(\Omega_{2}, \mathbb{R}^{M_{2}}\right)}}^{p_{2}} \\
& +\alpha \int_{\Omega_{1} \times \Omega_{1}} \frac{\left\|w_{n}(x)-w_{n}(y)\right\|_{\mathbb{R}^{M_{1}}}^{p_{1}}}{\|x-y\|_{\mathbb{R}^{N}}^{N+p_{1} s}} \rho^{l}(x-y) \mathrm{d}(x, y) \rightarrow+\infty .
\end{aligned}
$$

Case $2\left|w_{n}\right|_{W^{s, p_{1}}\left(\Omega_{1}, \mathbb{R}^{\left.M_{1}\right)}\right.} \rightarrow+\infty$.

If $l=0$, then $\mathcal{R}_{\left[\mathrm{d}_{1}\right]}$ is exactly the $W^{s, p_{1}}\left(\Omega_{1}, \mathbb{R}^{M_{1}}\right)$-seminorm $|w|_{W^{s, p_{1}}\left(\Omega_{1}, \mathbb{R}^{\left.M_{1}\right)}\right.}$ and we trivially get the desired result. Hence, we assume from now on that $l=1$. The assumptions on $\rho$ ensure that there exists a $\tau>0$ and $\eta_{\tau}>0$ such that

$$
\begin{aligned}
\mathcal{S}_{\tau}: & =\left\{(x, y) \in \Omega_{1} \times \Omega_{1}: \rho(x-y) \geq \tau\right\} \\
& =\left\{(x, y) \in \Omega_{1} \times \Omega_{1}:\|x-y\|_{\mathbb{R}^{N}} \leq \eta_{\tau}\right\},
\end{aligned}
$$

cf. Fig. 1.

Splitting $\Omega_{1} \times \Omega_{1}$ into $\mathcal{S}_{\tau}=: \mathcal{S}$ and its complement $\left(\Omega_{1} \times \Omega_{1}\right) \backslash \mathcal{S}_{\tau}=: \mathcal{S}^{c}$, we accordingly split the integrals $\left|w_{n}\right|_{W^{s, p_{1}}\left(\Omega_{1}, \mathbb{R}^{M_{1}}\right)}=\int_{\Omega_{1} \times \Omega_{1}} \frac{\left\|w_{n}(x)-w_{n}(y)\right\|_{\mathbb{R}^{M_{1}}}^{p_{1}}}{\|x-y\|_{\mathbb{R}^{N}}^{N+p_{1}{ }^{s}}} \mathrm{~d}(x, y)$ and consider again two cases $\int_{\mathcal{S}} \frac{\left\|w_{n}(x)-w_{n}(y)\right\|_{\mathbb{R}^{M_{1}}}^{p_{1}}}{\|x-y\|_{\mathbb{R}^{N}}^{N+p_{1} s}} \mathrm{~d}(x, y) \rightarrow+\infty$ and $\int_{\mathcal{S}^{c}} \frac{\left\|w_{n}(x)-w_{n}(y)\right\|_{\mathbb{R}^{M_{1}}}^{p_{1}}}{\|x-y\|_{\mathbb{R}^{N}}^{N+p_{1}{ }^{s}}} \mathrm{~d}(x, y) \rightarrow+\infty$, respectively.

Case 2.1 $\int_{\mathcal{S}} \frac{\left\|w_{n}(x)-w_{n}(y)\right\|_{\mathbb{R}^{M_{1}}}^{p_{1}}}{\|x-y\|_{\mathbb{R}^{N}}^{N+p_{1}}} \mathrm{~d}(x, y) \rightarrow+\infty$.

By definition of $\mathcal{S}$ we have $\rho(x-y) \geq \tau>0$ for all $(x, y) \in \mathcal{S}$. Therefore, 

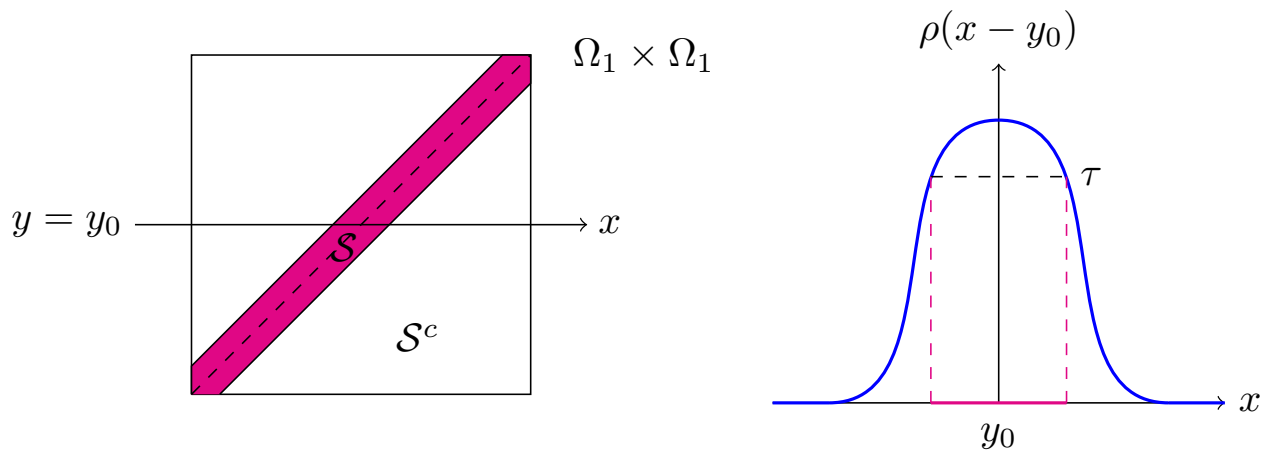

Fig. 1 The stripe $\mathcal{S}=\mathcal{S}_{\tau}$ if $\Omega_{1}$ is an open interval and its connection to the radial mollifier $\rho$ for fixed $y \in \Omega_{1}$

$$
\begin{aligned}
& \int_{\mathcal{S}} \frac{\left\|w_{n}(x)-w_{n}(y)\right\|_{\mathbb{R}^{M_{1}}}^{p_{1}}}{\|x-y\|_{\mathbb{R}^{N}}^{N+p_{1} s}} \rho(x-y) \mathrm{d}(x, y) \\
& \quad \geq \tau \int_{\mathcal{S}} \frac{\left\|w_{n}(x)-w_{n}(y)\right\|_{\mathbb{R}^{p_{1}}}^{p_{1}}}{\|x-y\|_{\mathbb{R}^{N}}^{N+p_{1} s}} \mathrm{~d}(x, y) \rightarrow+\infty .
\end{aligned}
$$

sider an arbitrarily chosen $w \in W^{s, p_{1}}\left(\Omega_{1}, K_{1}\right)$ fulfilling $\|\mathrm{F}(w)-v\|_{L^{p_{2}\left(\Omega_{2}, \mathbb{R}^{\left.M_{2}\right)}\right.}}^{p_{2}} \leq c$.

Then $\|\mathrm{F}(w)-v\|_{L^{p_{2}}\left(\Omega_{2}, \mathbb{R}^{M_{2}}\right)} \leq \sqrt[p_{2}]{c}$. Using the triangle inequality and the monotonicity of the function $h: t \mapsto t^{p_{2}}$ on $[0,+\infty)$, we get further

$$
\begin{aligned}
& \|\mathrm{F}(w)\|_{L^{p_{2}\left(\Omega_{2}, \mathbb{R}^{M_{2}}\right)}}^{p_{2}} \\
& =\|\mathrm{F}(w)-v+v\|_{L^{p_{2}}\left(\Omega_{2}, \mathbb{R}^{M_{2}}\right)}^{p_{2}}
\end{aligned}
$$

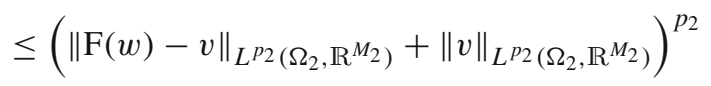

$$
\begin{aligned}
& \leq\left(\sqrt[p_{2}]{c}+\|v\|_{L^{p_{2}\left(\Omega_{2}, \mathbb{R}^{M_{2}}\right)}}\right)^{p_{2}}=: \tilde{c}
\end{aligned}
$$

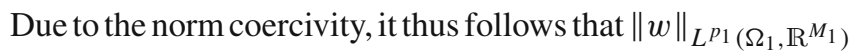
$\leq \bar{c}, \bar{c}$ some constant. Using [55, Lemma 3.20], it then follows that

$$
\begin{aligned}
& \|w(x)-w(y)\|_{\mathbb{R}^{M_{1}}}^{p_{1}} \leq 2^{p_{1}-1}\|w(x)\|_{\mathbb{R}^{M_{1}}}^{p_{1}} \\
& +2^{p_{1}-1}\|w(y)\|_{\mathbb{R}^{M_{1}}}^{p_{1}}
\end{aligned}
$$

for all $(x, y) \in \Omega_{1} \times \Omega_{1}$. Using Eq. 3.6, Fubini's Theorem and Eq. 3.5 we obtain

Case 2.2 $\int_{\mathcal{S}^{c}} \frac{\left\|w_{n}(x)-w_{n}(y)\right\|_{\mathbb{R}^{M_{1}}}^{p_{1}}}{\|x-y\|_{\mathbb{R}^{N}}^{N+p_{1}}} \mathrm{~d}(x, y) \rightarrow+\infty$.

For $(x, y) \in \mathcal{S}^{c}$ it might happen that $\rho(x-y)=0$, and thus instead of proving $\mathcal{F}\left(w_{n}\right) \geq \int_{\mathcal{S}^{c}} \frac{\left\|w_{n}(x)-w_{n}(y)\right\|_{\mathbb{R}^{M_{1}}}^{p_{1}}}{\|x-y\|_{\mathbb{R}^{N}}^{N+p_{1}}} \rho(x-$ $y) \mathrm{d}(x, y) \rightarrow+\infty$, as in Case 2.1, we rather show that $\mathcal{F}\left(w_{n}\right) \geq\left\|\mathrm{F}\left(w_{n}\right)-v\right\|_{L^{p_{2}\left(\Omega_{2}, \mathbb{R}^{\left.M_{2}\right)}\right.}}^{p_{2}} \rightarrow+\infty$. For this it is sufficient to show that for every $c>0$ there is some $C \in \mathbb{R}$ such that the implication

$$
\begin{aligned}
& \|\mathrm{F}(w)-v\|_{L^{p_{2}\left(\Omega_{2}, \mathbb{R}^{M_{2}}\right)}}^{p_{2}} \leq c \\
& \Longrightarrow \int_{\mathcal{S}^{c}} \frac{\|w(x)-w(y)\|_{\mathbb{R}^{M_{1}}}^{p_{1}}}{\|x-y\|_{\mathbb{R}^{N}}^{N+p_{1} s}} \mathrm{~d}(x, y) \leq C,
\end{aligned}
$$

holds true for all $w \in W^{s, p_{1}}\left(\Omega_{1}, K_{1}\right) \subseteq$ $W^{s, p_{1}}\left(\Omega_{1}, \mathbb{R}^{M_{1}}\right)$. To this end let $c>0$ be given and con- 
Combining $\|x-y\|_{\mathbb{R}^{N}} \geq \eta_{\tau}>0$ for all $(x, y) \in \mathcal{S}^{c}$ with the previous inequality, we obtain the needed estimate

$$
\begin{aligned}
& \int_{\mathcal{S}^{c}} \frac{\|w(x)-w(y)\|_{\mathbb{R}^{M_{1}}}^{p_{1}}}{\|x-y\|_{\mathbb{R}^{N}}^{N+p_{1} s}} \mathrm{~d}(x, y) \\
& \leq \frac{1}{\eta_{\tau}^{N+p_{1} s}} \int_{\mathcal{S}^{c}}\|w(x)-w(y)\|_{\mathbb{R}^{M_{1}}}^{p_{1}} \mathrm{~d}(x, y) \\
& \leq \frac{1}{\eta_{\tau}^{N+p_{1} s}} \int_{\Omega_{1} \times \Omega_{1}}\|w(x)-w(y)\|_{\mathbb{R}^{M_{1}}}^{p_{1}} \mathrm{~d}(x, y) \\
& \leq \frac{2^{p_{1}}\left|\Omega_{1}\right| \bar{c}^{p_{1}}}{\eta_{\tau}^{N+p_{1} s}}=: C .
\end{aligned}
$$

The second example concerns the coercivity of $\mathcal{F}\left[\mathrm{d}_{2}, \mathrm{~d}_{1}\right]$, defined in Eq. 2.9, when $\mathrm{F}$ denotes the masking operator occurring in image inpainting. To prove this result, we require the following auxiliary lemma:

Lemma 3.8 There exists a constant $C \in \mathbb{R}$ such that for all $w \in W^{s, p_{1}}\left(\Omega_{1}, \mathbb{R}^{M_{1}}\right), 0<s<1, l \in\{0,1\}, 1<p_{1}<\infty$ and $D \subsetneq \Omega_{1}$ nonempty such that

$$
\begin{aligned}
& \|w\|_{L^{p_{1}}\left(D, \mathbb{R}^{M_{1}}\right)}^{p_{1}} \\
& \leq C\left(\|w\|_{L^{p_{1}}\left(\Omega_{1} \backslash D, \mathbb{R}^{\left.M_{1}\right)}\right.}^{p_{1^{\prime}}}\right. \\
& \left.\quad+\int_{\Omega_{1} \times \Omega_{1}} \frac{\|w(x)-w(y)\|_{\mathbb{R}^{M_{1}}}^{p_{1}}}{\|x-y\|_{\mathbb{R}^{N}}^{N+p_{1} s}} \rho^{l}(x-y) \mathrm{d}(x, y)\right) .
\end{aligned}
$$

Proof The proof is inspired by the proof of Poincaré's inequality in [29]. It is included here for the sake of completeness.

Assume first that $l=1$. Let $\mathcal{S}$ be as above,

$$
\begin{aligned}
\mathcal{S} & :=\left\{(x, y) \in \Omega_{1} \times \Omega_{1}: \rho(x-y) \geq \tau\right\} \\
& =\left\{(x, y) \in \Omega_{1} \times \Omega_{1}:\|x-y\|_{\mathbb{R}^{N}} \leq \eta\right\} .
\end{aligned}
$$

If the stated inequality Eq. 3.7 would be false, then for every $n \in \mathbb{N}$ there would exists a function $w_{n} \in W^{s, p_{1}}\left(\Omega_{1}, \mathbb{R}^{M_{1}}\right)$ satisfying

$$
\begin{aligned}
\left\|w_{n}\right\|_{L^{p_{1}}\left(D, \mathbb{R}^{M_{1}}\right)}^{p_{1}} \geq & n\left(\left\|w_{n}\right\|_{L^{p_{1}}\left(\Omega_{1} \backslash D, \mathbb{R}^{M_{1}}\right)}^{p_{1}}\right. \\
& \left.+\int_{\Omega_{1} \times \Omega_{1}} \frac{\left\|w_{n}(x)-w_{n}(y)\right\|_{\mathrm{R}^{M_{1}}}^{p_{1}}}{\|x-y\|_{\mathrm{R}^{N}}^{N+p_{1} s}} \rho(x-y) \mathrm{d}(x, y)\right) .
\end{aligned}
$$

By normalizing we can assume without loss of generality (i) $\left\|w_{n}\right\|_{L^{p_{1}}\left(D, \mathbb{R}^{M_{1}}\right)}^{p_{1}}=1$.

Moreover, by Eq. 3.8

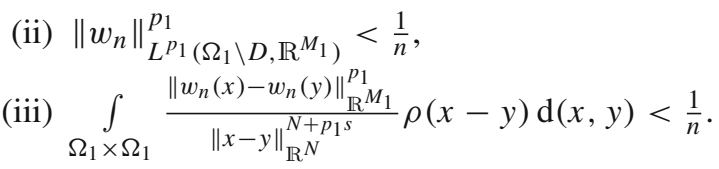

By item $(i)$ and item $(i i)$, we get that $\left\|w_{n}\right\|_{L^{p_{1}\left(\Omega_{1}, \mathbb{R}^{\left.M_{1}\right)}\right.}}^{p_{1}}=$ $\left\|w_{n}\right\|_{L^{p_{1}}\left(D, \mathbb{R}^{M_{1}}\right)}^{p_{1}}+\left\|w_{n}\right\|_{L^{p_{1}\left(\Omega_{1} \backslash D, \mathbb{R}^{\left.M_{1}\right)}\right.}}^{p_{1}}<1+\frac{1}{n}<2$ is bounded. Moreover

$$
\begin{aligned}
\left|w_{n}\right|_{W^{s, p_{1}}\left(\Omega_{1}, \mathbb{R}^{\left.M_{1}\right)}\right.}^{p_{1}} & =\int_{\mathcal{S}} \frac{\left\|w_{n}(x)-w_{n}(y)\right\|_{\mathbb{R}^{M_{1}}}^{p_{1}}}{\|x-y\|_{\mathbb{R}^{N}}^{N+p_{1} s}} \mathrm{~d}(x, y) \\
& +\int_{\mathcal{S}^{c}} \frac{\left\|w_{n}(x)-w_{n}(y)\right\|_{\mathbb{R}^{M_{1}}}^{p_{1}}}{\|x-y\|_{\mathbb{R}^{N}}^{N+p_{1} s}} \mathrm{~d}(x, y) \\
& \leq \frac{1}{\tau} \int_{\mathcal{S}} \frac{\left\|w_{n}(x)-w_{n}(y)\right\|_{\mathbb{R}^{M_{1}}}^{p_{1}}}{\|x-y\|_{\mathbb{R}^{N}}^{N+p_{1} s}} \rho(x-y) \mathrm{d}(x, y) \\
& +\frac{2_{1}^{p}\left|\Omega_{1}\right|}{\eta^{N+p_{1} s}}\left\|w_{n}\right\|_{L^{p_{1}}\left(\Omega_{1}, \mathbb{R}^{M_{1}}\right)}^{p_{1}} \\
& <\frac{1}{\tau n}+\frac{2^{p_{1}+1}\left|\Omega_{1}\right|}{\eta^{N+p_{1} s}} \\
& \leq \frac{1}{\tau}+\frac{2^{p_{1}+1}\left|\Omega_{1}\right|}{\eta^{N+p_{1} s}}=: c<\infty,
\end{aligned}
$$

where $c$ is independent of $n$. This yields that the sequence $\left(w_{n}\right)_{n \in \mathbb{N}}$ is bounded in $W^{s, p_{1}}\left(\Omega_{1}, \mathbb{R}^{M_{1}}\right)$ by $(2+c)^{\frac{1}{p_{1}}}$. By the reflexivity of $W^{s, p_{1}}\left(\Omega_{1}, \mathbb{R}^{M_{1}}\right)$ for $p_{1} \in(1, \infty)$ and Lemma 2.8 , there exists a subsequence $\left(w_{n_{k}}\right)_{k \in \mathbb{N}}$ of $\left(w_{n}\right)_{n \in \mathbb{N}}$ and $w_{*} \in W^{s, p_{1}}\left(\Omega_{1}, \mathbb{R}^{M_{1}}\right)$ such that $w_{n_{k}} \rightarrow w^{*}$ strongly in $L^{p_{1}}\left(\Omega_{1}, \mathbb{R}^{M_{1}}\right)$ and pointwise almost everywhere.

Using the continuity of the norm and dominated convergence, we obtain

(i) $\left\|w^{*}\right\|_{L^{p_{1}}\left(D, \mathbb{R}^{\left.M_{1}\right)}\right.}^{p_{1}}=1$, in particular, $w^{*}$ is not the null function on $\mathrm{D}$,

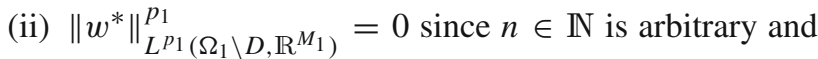
hence $w^{*} \equiv 0$ on $\Omega_{1} \backslash D$.

(iii)

$$
\begin{aligned}
& \liminf _{n \rightarrow \infty} \frac{1}{n} \\
& \geq \liminf _{n \rightarrow \infty} \int_{\mathcal{S}} \frac{\left\|w_{n}(x)-w_{n}(y)\right\|_{\mathbb{R}^{M_{1}}}^{p_{1}}}{\|x-y\|_{\mathbb{R}^{N}}^{N+p_{1}}} \rho(x-y) \mathrm{d}(x, y) \\
& \geq \frac{\tau}{\eta^{N+p_{1} s}} \int_{\mathcal{S}}\left\|w^{*}(x)-w^{*}(y)\right\|_{\mathbb{R}^{M_{1}}}^{p_{1}},
\end{aligned}
$$


i.e., $w^{*}(x)=w^{*}(y)$ for $(x, y) \in \mathcal{S}$ yielding that $w^{*}$ locally constant and hence even constant since $\Omega_{1}$ is connected,

which gives the contradiction.

In the case $l=0$ we use similar arguments, where the distance $\|x-y\|_{\mathbb{R}^{N}}$ in the last inequality can be estimated by diam $\left|\Omega_{1}\right|$ (instead of $\eta$ ) since $\Omega_{1}$ is bounded.

Remark 3.9 In case $l=1$ it follows that the sharper inequality holds true: There exists a constant $C \in \mathbb{R}$ such that for all $w \in W^{s, p_{1}}\left(\Omega_{1}, \mathbb{R}^{M_{1}}\right), 0<s<1,1<p_{1}<\infty$ and $D \subsetneq \Omega_{1}$ nonempty such that

$$
\begin{aligned}
& \|w\|_{L^{p_{1}}\left(D, \mathbb{R}^{M_{1}}\right)}^{p_{1}} \\
& \leq C\left(\|w\|_{L^{p_{1}}\left(\Omega_{1} \backslash D, \mathbb{R}^{\left.M_{1}\right)}\right.}^{p_{1}}\right. \\
& \left.+\int_{\mathcal{S}} \frac{\|w(x)-w(y)\|_{\mathbb{R}^{M_{1}}}^{p_{1}}}{\|x-y\|_{\mathbb{R}^{N}}^{N+p_{1} s}} \rho^{l}(x-y) \mathrm{d}(x, y)\right) .
\end{aligned}
$$

Example 3.10 As in Example 3.7, we consider that $W\left(\Omega_{1}, K_{1}\right)=W^{s, p_{1}}\left(\Omega_{1}, K_{1}\right)$ with $p_{1}>1,0<s<1$ and fix $k=N$.

Assume that $\mathrm{F}$ is the inpainting operator, i.e.,

$\mathrm{F}(w)=\chi_{\Omega_{1} \backslash D}(w)$,

where $D \subseteq \Omega_{1}, w \in W^{s, p_{1}}\left(\Omega_{1}, K_{1}\right)$. Since the dimension of the data $w$ and the image data $\mathrm{F}(w)$ has the same dimension at every point $x \in \Omega_{1}$, we write $M:=M_{1}=M_{2}$.

Then the functional $\mathcal{F}\left[\mathrm{d}_{2}, \mathrm{~d}_{1}\right]: W^{s, p_{1}}\left(\Omega_{1}, K_{1}\right) \rightarrow$ $[0, \infty]$ :

$\mathcal{F}\left[\mathrm{d}_{2}, \mathrm{~d}_{1}\right]=\llbracket \mathrm{F}(w), v \rrbracket_{\left[\mathrm{d}_{2}\right]}^{p_{2}}+\alpha \mathcal{R}_{\left[\mathrm{d}_{1}\right]}(w)$

is coercive for $p_{2} \geq p_{1}$ :

The fact that $p_{2} \geq p_{1}$ and that $\Omega_{1}$ is bounded ensures that

$L^{p_{2}}\left(\Omega_{1} \backslash D, \mathbb{R}^{M}\right) \subseteq L^{p_{1}}\left(\Omega_{1} \backslash D, \mathbb{R}^{M}\right)$.

The proof is done using the same arguments as in the proof of Example 3.7, where we additionally split Case 1 into the two subcases

Case $1.1\left\|w_{n}\right\|_{L^{p_{1}\left(D, \mathbb{R}^{M}\right)}} \rightarrow+\infty$

Case $1.2\left\|w_{n}\right\|_{L^{p_{1}}\left(\Omega_{1} \backslash D, \mathbb{R}^{M}\right)} \rightarrow+\infty$

and using additionally Lemma 3.8, Eqs. 3.9 and 3.10.

\section{Stability and Convergence}

In this section we will first show a stability and afterwards a convergence result. We use the notation introduced in Sect. 2. In particular, $W\left(\Omega_{1}, K_{1}\right)$ is as defined in Eq. 2.5. We also stress that we use notationally simplified versions $\mathcal{F}^{v}$ of $\mathcal{F}_{\alpha}^{v}\left[\mathrm{~d}_{2}, \mathrm{~d}_{1}\right]$ and $\mathcal{R}$ of $\mathcal{R}_{\left[\mathrm{d}_{1}\right]}$ whenever possible. See Eqs. 2.6, 2.7 and 2.8 .

Theorem 4.1 Let Assumption 3.2 be satisfied. Let $v^{\delta} \in$ $L^{p_{2}}\left(\Omega_{2}, K_{2}\right)$ and let $\left(v_{n}\right)_{n \in \mathbb{N}}$ be a sequence in $L^{p_{2}}\left(\Omega_{2}, K_{2}\right)$ such that $\llbracket v_{n}, v^{\delta} \rrbracket_{\left[\mathrm{d}_{2}\right]} \rightarrow 0$. Then every sequence $\left(w_{n}\right)_{n \in \mathbb{N}}$ with

$w_{n} \in \arg \min \left\{\mathcal{F}_{\alpha}^{v_{n}}\left[\mathrm{~d}_{2}, \mathrm{~d}_{1}\right](w): w \in W\left(\Omega_{1}, K_{1}\right)\right\}$

has a converging subsequence w.r.t. the topology of $W\left(\Omega_{1}, K_{1}\right)$. The limit $\tilde{w}$ of any such converging subsequence $\left(w_{n_{k}}\right)_{k \in \mathbb{N}}$ is a minimizer of $\mathcal{F}^{v^{\delta}}\left[\mathrm{d}_{2}, \mathrm{~d}_{1}\right]$. Moreover, $\left(\mathcal{R}\left(w_{n_{k}}\right)\right)_{k \in \mathbb{N}}$ converges to $\mathcal{R}(\tilde{w})$.

The subsequent proof of Theorem 4.1 is similar to the proof of [55, Theorem 3.23].

Proof For the ease of notation, we simply write $\mathcal{F}^{v^{\delta}}$ instead of $\mathcal{F}_{\alpha}^{v^{\delta}}\left[\mathrm{d}_{2}, \mathrm{~d}_{1}\right]$ and $\llbracket v, \tilde{v} \rrbracket=\llbracket v, \tilde{v} \rrbracket_{\left[\mathrm{d}_{2}\right]}$.

By assumption the sequence $\left(\llbracket v_{n}, v^{\delta} \rrbracket\right)_{n \in \mathbb{N}}$ converges to 0 and thus is bounded, i.e., there exists $B \in(0,+\infty)$ such that

$\llbracket v_{n}, v^{\delta} \rrbracket \leq B$ for all $n \in \mathbb{N}$.

Because $w_{n} \in \arg \min \left\{\mathcal{F}^{v_{n}}(w): w \in W\left(\Omega_{1}, K_{1}\right)\right\}$ it follows that

$\mathcal{F}^{v_{n}}\left(w_{n}\right) \leq \mathcal{F}^{v_{n}}(w)$ for all $w \in W\left(\Omega_{1}, K_{1}\right)$

By Assumption 3.2 there is a $\bar{w} \in W\left(\Omega_{1}, K_{1}\right)$ such that $\mathcal{F}^{v^{0}}(\bar{w})<\infty$. Set $c:=2^{p_{2}-1}$. Using Assumption 3.2 and applying Lemma 3.5, Eqs. 4.2 and 4.1 implies that for all $n \in \mathbb{N}$

$$
\begin{aligned}
\mathcal{F}^{v^{\delta}}\left(w_{n}\right) & \leq c \mathcal{F}^{v_{n}}\left(w_{n}\right)+c \llbracket v_{n}, v^{\delta} \rrbracket^{p_{2}} \\
& \leq c \mathcal{F}^{v_{n}}(\bar{w})+c B^{p_{2}} \\
& \leq c\left[c \mathcal{F}^{v^{\delta}}(\bar{w})+c \llbracket v^{\delta}, v_{n} \rrbracket^{p_{2}}\right]+c B^{p_{2}} \\
& \leq c^{2} \mathcal{F}^{v^{\delta}}(\bar{w})+\left(c^{2}+c\right) B^{p_{2}} \\
\leq & c^{3}\left(\mathcal{F}^{v^{0}}(\bar{w})+\llbracket v^{0}, v^{\delta} \rrbracket\right) \\
& +\left(c^{2}+c\right) B^{p_{2}}=: m<\infty .
\end{aligned}
$$

Applying again Lemma 3.5, we obtain $\mathcal{F}^{v^{0}}\left(w_{n}\right) \leq c \mathcal{F}^{v^{\delta}}\left(w_{n}\right)+$ $c \llbracket v^{\delta}, v^{0} \rrbracket^{p_{2}} \leq m+c \llbracket v^{\delta}, v^{0} \rrbracket^{p_{2}}=: \tilde{m}<\infty$. Hence, from 
item (3.1) it follows that the sequence $\left(w_{n}\right)_{n \in \mathbb{N}}$ contains a converging subsequence.

Let now $\left(w_{n_{k}}\right)_{k \in \mathbb{N}}$ be an arbitrary subsequence of $\left(w_{n}\right)_{n \in \mathbb{N}}$ which converges in $W\left(\Omega_{1}, K_{1}\right)$ to some $\tilde{w} \in W\left(\Omega_{1}, \mathbb{R}^{M_{1}}\right)$. Then, from Lemma 2.8 and the continuity properties of $\mathrm{F}$ it follows that $\tilde{w} \in W\left(\Omega_{1}, K_{1}\right)$ and $\left(\mathrm{F}\left(w_{n_{k}}\right), v_{n_{k}}\right) \rightarrow$ $\left(\mathrm{F}(\tilde{w}), v^{\delta}\right)$ in $L^{p_{2}}\left(\Omega_{2}, K_{2}\right) \times L^{p_{2}}\left(\Omega_{2}, K_{2}\right)$. Moreover, using Lemma 3.4, Eq. 4.2 and the triangle inequality it follows that for every $w \in W\left(\Omega_{1}, K_{1}\right)$ the following estimate holds true

$$
\begin{aligned}
\mathcal{F}^{v^{\delta}}(\tilde{w}) & =\llbracket \mathrm{F}(\tilde{w}), v^{\delta} \rrbracket^{p_{2}}+\alpha \mathcal{R}(\tilde{w}) \\
\leq & \llbracket \mathrm{F}(\tilde{w}), v^{\delta} \rrbracket^{p_{2}}+\alpha \liminf _{k \rightarrow \infty} \mathcal{R}\left(w_{n_{k}}\right) \\
& \leq \llbracket \mathrm{F}(\tilde{w}), v^{\delta} \rrbracket^{p_{2}}+\alpha \limsup _{k \rightarrow \infty} \mathcal{R}\left(w_{n_{k}}\right) \\
& \leq \liminf _{k \rightarrow \infty} \llbracket \mathrm{F}\left(w_{n_{k}}\right), v_{n_{k}} \rrbracket^{p_{2}}+\alpha \limsup _{k \rightarrow \infty} \mathcal{R}\left(w_{n_{k}}\right) \\
& \leq \limsup _{k \rightarrow \infty} \mathcal{F}^{v_{n_{k}}}\left(w_{n_{k}}\right) \\
& \leq \limsup _{k \rightarrow \infty} \mathcal{F}^{v_{n_{k}}}(w) \\
& =\left(\limsup _{k \rightarrow \infty} \llbracket F(w), v_{n_{k}} \rrbracket\right)^{p_{2}}+\alpha \mathcal{R}(w) \\
\leq & \left(\limsup _{k \rightarrow \infty}\left(\llbracket F(w), v^{\delta} \rrbracket+\llbracket v^{\delta}, v_{n_{k}} \rrbracket\right)\right)^{p_{2}}+\alpha \mathcal{R}(w) \\
& =\limsup _{k \rightarrow \infty}\left(\llbracket \mathrm{F}(w), v_{n_{k}} \rrbracket^{p_{2}}+\alpha \mathcal{R}(w)\right) \\
& =\llbracket \mathrm{F}(w), v^{\delta} \rrbracket^{p_{2}}+\alpha \mathcal{R}(w) \\
& =\mathcal{F}^{v^{\delta}}(w) .
\end{aligned}
$$

This shows that $\tilde{w}$ is a minimizer of $\mathcal{F}^{v^{\delta}}$. Choosing $w=\tilde{w}$ in the previous estimate, we obtain the equality

$$
\begin{aligned}
& \llbracket \mathrm{F}(\tilde{w}), v^{\delta} \rrbracket^{p_{2}}+\alpha \mathcal{R}(\tilde{w}) \\
& =\llbracket \mathrm{F}(\tilde{w}), v^{\delta} \rrbracket^{p_{2}}+\alpha \liminf _{k \rightarrow \infty} \mathcal{R}\left(w_{n_{k}}\right) \\
& =\llbracket \mathrm{F}(\tilde{w}), v^{\delta} \rrbracket^{p_{2}}+\alpha \limsup _{k \rightarrow \infty} \mathcal{R}\left(w_{n_{k}}\right) .
\end{aligned}
$$

Due to $\llbracket \mathrm{F}(\tilde{w}), v^{\delta} \rrbracket^{p_{2}} \leq \mathcal{F}^{v^{\delta}}(\tilde{w}) \leq m<\infty$ this gives

$\mathcal{R}(\tilde{w})=\lim _{k \rightarrow \infty} \mathcal{R}\left(w_{n_{k}}\right)$.

Before proving the next theorem, we need the following definition, cf. [55].

Definition 4.2 Let $v^{0} \in L^{p_{2}}\left(\Omega_{2}, K_{2}\right)$. Every element $w^{*} \in$ $W\left(\Omega_{1}, K_{1}\right)$ fulfilling

$$
\begin{aligned}
& \mathrm{F}\left(w^{*}\right)=v^{0} \\
& \mathcal{R}\left(w^{*}\right)=\min \left\{\mathcal{R}(w): w \in W\left(\Omega_{1}, K_{1}\right), \mathrm{F}(w)=v^{0}\right\} .
\end{aligned}
$$

is called an $\mathcal{R}$-minimizing solution of the equation $\mathrm{F}(w)=v^{0}$ or shorter just $\mathcal{R}$-minimizing solution.

The following theorem and its proof are inspired by [55, Theorem 3.26].

Theorem 4.3 Let Assumption 3.2 be satisfied. Let there exist an $\mathcal{R}$-minimizing solution $w^{\dagger} \in W\left(\Omega_{1}, K_{1}\right)$ and let $\alpha$ : $(0, \infty) \rightarrow(0, \infty)$ be a function satisfying

$\alpha(\delta) \rightarrow 0$ and $\frac{\delta^{p_{2}}}{\alpha(\delta)} \rightarrow 0$ for $\delta \rightarrow 0$

Let $\left(\delta_{n}\right)_{n \in \mathbb{N}}$ be a sequence of positive real numbers converging to 0 . Moreover, let $\left(v_{n}\right)_{n \in \mathbb{N}}$ be a sequence in $L^{p_{2}}\left(\Omega_{2}, K_{2}\right)$ with $\llbracket v^{0}, v_{n} \rrbracket_{\left[\mathrm{d}_{2}\right]} \leq \delta_{n}$ and set $\alpha_{n}:=\alpha\left(\delta_{n}\right)$.

Then every sequence $\left(w_{n}\right)_{n \in \mathbb{N}}$ of minimizers

$w_{n} \in \arg \min \left\{\mathcal{F}_{\alpha_{n}}^{v_{n}}\left[\mathrm{~d}_{2}, \mathrm{~d}_{1}\right](w): w \in W\left(\Omega_{1}, K_{1}\right)\right\}$

has a converging subsequence $w_{n_{k}} \stackrel{W}{\rightarrow} \tilde{w}$ as $k \rightarrow \infty$, and the limit $\tilde{w}$ is always an $\mathcal{R}$-minimizing solution. In addition, $\mathcal{R}\left(w_{n_{k}}\right) \rightarrow \mathcal{R}(\tilde{w})$.

Moreover, if $w^{\dagger}$ is unique, it follows that $w_{n} \stackrel{W}{\rightarrow} w^{\dagger}$ and $\mathcal{R}\left(w_{n}\right) \rightarrow \mathcal{R}\left(w^{\dagger}\right)$.

Proof We write shortly $\llbracket \cdot, \cdot \rrbracket$ for $\llbracket \cdot, \cdot \rrbracket_{\left[\mathrm{d}_{2}\right]}$. Taking into account that $w_{n} \in \operatorname{argmin}\left\{\mathcal{F}_{\alpha_{n}}^{v_{n}}\left[\mathrm{~d}_{2}, \mathrm{~d}_{1}\right](w): w \in W\left(\Omega_{1}, K_{1}\right)\right\}$ it follows that

$$
\begin{aligned}
& \llbracket \mathrm{F}\left(w_{n}\right), v_{n} \rrbracket^{p_{2}} \leq \mathcal{F}_{\alpha_{n}}^{v_{n}}\left(w_{n}\right) \leq \mathcal{F}_{\alpha_{n}}^{v_{n}}\left(w^{\dagger}\right) \\
& \quad=\llbracket v^{0}, v_{n} \rrbracket^{p_{2}}+\alpha_{n} \mathcal{R}\left(w^{\dagger}\right) \leq \delta_{n}^{p_{2}}+\alpha_{n} \mathcal{R}\left(w^{\dagger}\right) \rightarrow 0,
\end{aligned}
$$

yielding $\llbracket \mathrm{F}\left(w_{n}\right), v_{n} \rrbracket \rightarrow 0$ as $n \rightarrow \infty$. The triangle inequality gives $\llbracket \mathrm{F}\left(w_{n}\right), v^{0} \rrbracket \leq \llbracket \mathrm{F}\left(w_{n}\right), v_{n} \rrbracket+\llbracket v_{n}, v^{0} \rrbracket \rightarrow 0$ as $n \rightarrow$ $\infty$ and Remark 3.1 ensures $\left\|F\left(w_{n}\right)-v^{0}\right\|_{L^{p_{2}\left(\Omega_{2}, \mathbb{R}^{M_{2}}\right)}} \leq$ $\llbracket \mathrm{F}\left(w_{n}\right), v^{0} \rrbracket \rightarrow 0$ as $n \rightarrow \infty$, so that

$\mathrm{F}\left(w_{n}\right) \rightarrow v^{0}$ in $L^{p_{2}}\left(\Omega_{2}, \mathbb{R}^{M_{2}}\right)$.

Since

$$
\begin{aligned}
& \mathcal{R}\left(w_{n}\right) \leq \frac{1}{\alpha_{n}} \mathcal{F}_{\alpha_{n}}^{v_{n}}\left(w_{n}\right) \leq \frac{1}{\alpha_{n}} \mathcal{F}_{\alpha_{n}}^{v_{n}}\left(w^{\dagger}\right) \\
& =\frac{1}{\alpha_{n}}\left(\llbracket v^{0}, v_{n} \rrbracket^{p_{2}}+\alpha_{n} \mathcal{R}\left(w^{\dagger}\right)\right) \leq \frac{\delta_{n}^{p_{2}}}{\alpha_{n}}+\mathcal{R}\left(w^{\dagger}\right),
\end{aligned}
$$

we also get

$$
\limsup _{n \rightarrow \infty} \mathcal{R}\left(w_{n}\right) \leq \mathcal{R}\left(w^{\dagger}\right)
$$

Set $\alpha_{\max }:=\max \left\{\alpha_{n}: n \in \mathbb{N}\right\}$. Since 


$$
\begin{aligned}
\limsup _{n \rightarrow \infty} \mathcal{F}_{\alpha_{n}}^{v^{0}}\left(w_{n}\right) & \leq \limsup _{n \rightarrow \infty}\left(\llbracket \mathrm{F}\left(w_{n}\right), v^{0} \rrbracket^{p_{2}}+\alpha_{\max } \mathcal{R}\left(w_{n}\right)\right) \\
& \leq \alpha_{\max } \mathcal{R}\left(w^{\dagger}\right)
\end{aligned}
$$

the sequence $\mathcal{F}_{\alpha_{\max }}^{v^{0}}\left(w_{n}\right)$ is bounded. From Assumption 3.2, item (3.1) it follows that there exists a converging subsequence $\left(w_{n_{k}}\right)_{k \in \mathbb{N}}$ of $\left(w_{n}\right)_{n \in \mathbb{N}}$. The limit of $\left(w_{n_{k}}\right)_{k \in \mathbb{N}}$ is denoted by $\tilde{w}$. Then, from Lemma 2.8 it follows that $\tilde{w} \in$ $W\left(\Omega_{1}, K_{1}\right)$. Since the operator $\mathrm{F}$ is sequentially continuous, it follows that $\mathrm{F}\left(w_{n_{k}}\right) \rightarrow \mathrm{F}(\tilde{w})$ in $L^{p_{2}}\left(\Omega_{2}, K_{2}\right)$. This shows that actually $\mathrm{F}(\tilde{w})=v^{0}$ since Eq. 4.5 is valid. Then, from Lemma 3.4 it follows that the functional $\mathcal{R}: W\left(\Omega_{1}, K_{1}\right) \rightarrow$ $[0,+\infty]$ is sequentially lower semi-continuous, so that $\mathcal{R}(\tilde{w}) \leq \liminf _{k \rightarrow \infty} \mathcal{R}\left(w_{n_{k}}\right)$. Combining this with Eq. 4.6, we also obtain

$$
\begin{aligned}
\mathcal{R}(\tilde{w}) & \leq \liminf _{k \rightarrow \infty} \mathcal{R}\left(w_{n_{k}}\right) \leq \limsup _{k \rightarrow \infty} \mathcal{R}\left(w_{n_{k}}\right) \leq \mathcal{R}\left(w^{\dagger}\right) \\
& \leq \mathcal{R}(\tilde{w}),
\end{aligned}
$$

using the definition of $w^{\dagger}$. This, together with the fact that $\mathrm{F}(\tilde{w})=v^{0}$ we see that $\tilde{w}$ is an $\mathcal{R}$-minimizing solution and that $\lim _{k \rightarrow \infty} \mathcal{R}\left(w_{n_{k}}\right)=\mathcal{R}(\tilde{w})$.

Now assume that the solution fulfilling Eq. 4.3 is unique; we call it $w^{\dagger}$. In order to prove that $w_{n} \stackrel{W}{\rightarrow} w^{\dagger}$, it is sufficient to show that any subsequence has a further subsequence converging to $w^{\dagger}$, cf. [55, Lemma 8.2]. Hence, denote by $\left(w_{n_{k}}\right)_{k \in \mathbb{N}}$ an arbitrary subsequence of $\left(w_{n}\right)$, the sequence of minimizers. Like before we can show that $\mathcal{F}_{\alpha}^{v^{0}}\left(w_{n_{k}}\right)$ is bounded and we can extract a converging subsequence $\left(w_{n_{k_{l}}}\right)_{l \in \mathbb{N}}$. The limit of this subsequence is $w^{\dagger}$ since it is the unique solution fulfilling Eq. 4.3, showing that $w_{n} \stackrel{W}{\rightarrow}$ $w^{\dagger}$. Moreover, $w^{\dagger} \in W\left(\Omega_{1}, K_{1}\right)$. Following the arguments above, we obtain as well $\lim _{n \rightarrow \infty} \mathcal{R}\left(w_{n}\right)=\mathcal{R}\left(w^{\dagger}\right)$.

Remark 4.4 Theorem 4.1 guarantees that the minimizers of $\mathcal{F}_{\alpha}^{v_{n}}\left[\mathrm{~d}_{2}, \mathrm{~d}_{1}\right]$ depend continuously on $v^{\delta}$, while Theorem 4.3 ensures that they converge to a solution of $\mathrm{F}(w)=v^{0}, v^{0}$ the exact data, while $\alpha$ tends to zero.

\section{Discussion of the Results and Conjectures}

In this section we summarize some open problems related to double integral expressions of functions with values on manifolds.

\subsection{Relation to Single Integral Representations}

In the following we show for one particular case of functions that have values in a manifold, that the double integral formulation $\mathcal{R}_{\left[\mathrm{d}_{1}\right]}$, defined in Eq. 2.8, approximates a single energy integral. The basic ingredient for this derivation is the exponential map related to the metric $d_{1}$ on the manifold. In the following we investigate manifold-valued functions $w \in W^{1,2}(\Omega, \mathcal{M})$, where we consider $\mathcal{M} \subseteq \mathbb{R}^{M \times 1}$ to be a connected, complete Riemannian manifold. In this case some of the regularization functionals $\mathcal{R}_{\left[\mathrm{d}_{1}\right]}$, defined in Eq. 2.8, can be considered as approximations of single integrals. In particular, we aim to generalize Eq. 1.3 in the case $p=2$.

We have that

$\nabla w=\left[\begin{array}{ccc}\frac{\partial w_{1}}{\partial x_{1}} & \cdots & \frac{\partial w_{1}}{\partial x_{N}} \\ \vdots & \ddots & \vdots \\ \frac{\partial w_{M}}{\partial x_{1}} & \cdots & \frac{\partial w_{M}}{\partial x_{N}}\end{array}\right] \in \mathbb{R}^{M \times N}$

In the following we will write $\mathcal{R}_{\left[\mathrm{d}_{1}\right], \varepsilon}$ instead of $\frac{1}{2} \mathcal{R}_{\mathrm{d}_{1}}$ to stress the dependence on $\varepsilon$ in contrast to above; the factor $\frac{1}{2}$ was added due to reasons of calculation. Moreover, let $\hat{\rho}: \mathbb{R}_{+} \rightarrow \mathbb{R}_{+}$be in $C_{c}^{\infty}\left(\mathbb{R}_{+}, \mathbb{R}_{+}\right)$and satisfy

$\left|\mathbb{S}^{N-1}\right| \int_{0}^{\infty} \hat{t}^{N-1} \hat{\rho}(\hat{t}) d \hat{t}=1$

Then for every $\varepsilon>0$

$x \in \mathbb{R}^{n} \mapsto \rho_{\varepsilon}(x):=\frac{1}{\varepsilon^{N}} \hat{\rho}\left(\frac{\|x\|_{\mathbb{R}^{N}}}{\varepsilon}\right)$

is a mollifier, cf. Example 2.2.

$\mathcal{R}_{\left[\mathrm{d}_{1}\right], \varepsilon}$ (with $p_{1}=2$ ) then reads as follows:

$\mathcal{R}_{\left[\mathrm{d}_{1}\right], \varepsilon}(w):=\frac{1}{2} \int_{\Omega \times \Omega} \frac{d_{1}^{2}(w(x), w(y))}{\|x-y\|_{\mathbb{R}^{N}}^{2}} \rho_{\varepsilon}(x-y) \mathrm{d}(x, y)$.

Substitution with spherical coordinates $y=x-t \theta \in \mathbb{R}^{N \times 1}$ with $\theta \in \mathbb{S}^{N-1} \subseteq \mathbb{R}^{N \times 1}, t \geq 0$ gives

$$
\begin{aligned}
& \lim _{\varepsilon \searrow 0} \mathcal{R}_{\left[\mathrm{d}_{1}\right], \varepsilon}(w) \\
& =\lim _{\varepsilon \searrow 0} \frac{1}{\varepsilon^{N}} \int_{\Omega} \int_{\mathbb{S}^{N-1}} \int_{0}^{\infty} \frac{1}{2} d_{1}^{2}(w(x), w(x-t \theta)) t^{N-3} \hat{\rho}\left(\frac{t}{\varepsilon}\right) \mathrm{d} t \mathrm{~d} \theta \mathrm{d} x .
\end{aligned}
$$

Now, using that for $m_{1} \in \mathcal{M}$ fixed and $m_{2} \in \mathcal{M}$ such that $m_{1}$ and $m_{2}$ are joined by a unique minimizing geodesic (see, for instance, [30] where the concept of exponential mappings is explained)

$$
\frac{1}{2} \partial_{2} d_{1}^{2}\left(m_{1}, m_{2}\right)=-\left(\exp _{m_{2}}\right)^{-1}\left(m_{1}\right) \in \mathbb{R}^{M \times 1},
$$


where $\partial_{2}$ denotes the derivative of $d_{1}^{2}$ with respect to the second component. By application of the chain rule we get

$$
\begin{aligned}
- & \frac{1}{2} \nabla_{y} d_{1}^{2}(w(x), w(y)) \\
= & \underbrace{(\nabla w(y))^{\mathrm{T}}}_{\in \mathbb{R}^{N \times M}} \underbrace{\left(\exp _{w(y)}\right)^{-1}(w(x))}_{\in \mathbb{R}^{M \times 1}} \in \mathbb{R}^{N \times 1},
\end{aligned}
$$

where $w(x)$ and $w(y)$ are joined by a unique minimizing geodesic. This assumption seems reasonable due to the fact that we consider the case $\varepsilon \searrow 0$. Let - denote the scalar multiplication of two vectors in $\mathbb{R}^{N \times 1}$, then the last equality shows that

$$
\begin{aligned}
\frac{1}{2} d_{1}^{2}(w(x), w(x-t \theta)) \\
=-\frac{1}{2}\left[d_{1}^{2}(w(x), w((x-t \theta)+t \theta))\right. \\
\left.\quad-d_{1}^{2}(w(x), w(x-t \theta))\right] \\
\approx\left((\nabla w(x-t \theta))^{\mathrm{T}}\left(\exp _{w(x-t \theta)}\right)^{-1}(w(x))\right) \cdot t \theta .
\end{aligned}
$$

Thus, from Eq. 5.2 it follows that

$$
\begin{aligned}
\lim _{\varepsilon \searrow 0} & \mathcal{R}_{\left[\mathrm{d}_{1}\right], \varepsilon}(w) \\
\approx & \lim _{\varepsilon \searrow 0} \frac{1}{\varepsilon^{N}} \int_{\Omega} \int_{\mathbb{S}^{N-1}} \int_{0}^{\infty}\left((\nabla w(x-t \theta))^{\mathrm{T}}\left(\exp _{w(x-t \theta)}\right)^{-1}(w(x))\right) \\
& \cdot \theta\left(t^{N-2} \hat{\rho}\left(\frac{t}{\varepsilon}\right)\right) \mathrm{d} t \mathrm{~d} \theta \mathrm{d} x
\end{aligned}
$$

Now we will use a Taylor series of power 0 for $t \mapsto \nabla w(x-$ $t \theta)$ and of power 1 for $t \mapsto\left(\exp _{w(x-t \theta)}\right)^{-1}(w(x))$ to rewrite Eq. 5.4. We write

$$
F(w ; x, t, \theta):=\left(\exp _{w(x-t \theta)}\right)^{-1}(w(x)) \in \mathbb{R}^{M \times 1}
$$

and define

$$
\begin{aligned}
\dot{F}(w ; x, \theta):=\lim _{t \searrow 0} \frac{1}{t}\left(\left(\exp _{w(x-t \theta)}\right)^{-1}(w(x))\right. \\
-\underbrace{\left(\exp _{w(x)}\right)^{-1}(w(x))}_{=0}) \in \mathbb{R}^{M \times 1} .
\end{aligned}
$$

Note that because $\left(\exp _{w(x)}\right)^{-1}(w(x))$ vanishes, $\dot{F}(w(x) ; \theta)$ is the leading order term of the expansion of $\left(\exp _{w(x-t \theta)}\right)^{-1}$ $(w(x))$ with respect to $t$. Moreover, in the case that $\nabla w(x) \neq$ 0 this is the leading order approximation of $\nabla w(x-t \theta)$. In summary we are calculating the leading order term of the expansion with respect to $t$.
Then from Eq. 5.4 it follows that

$$
\begin{aligned}
& \lim _{\varepsilon \searrow 0} \mathcal{R}_{\left[\mathrm{d}_{1}\right], \varepsilon}(w) \approx \lim _{\varepsilon \searrow 0} \underbrace{\frac{1}{\varepsilon^{N}} \int_{0}^{\infty} t^{N-1} \hat{\rho}\left(\frac{t}{\varepsilon}\right) \mathrm{d} t}_{=\left|\mathbb{S}^{N-1}\right|^{-1}} \\
& \quad \times \int_{\Omega} \int_{\mathbb{S}^{N-1}}\left((\nabla w(x))^{\mathrm{T}} \dot{F}(w ; x, \theta)\right) \cdot \theta \mathrm{d} \theta \mathrm{d} x .
\end{aligned}
$$

The previous calculations show that the double integral simplifies to a double integral where the inner integration domain has one dimension less than the original integral. Under certain assumption the integration domain can be further simplified:

Example 5.1 If $d_{1}(x, y)=\|x-y\|_{\mathbb{R}^{M}}, p_{1}=2$, then

$$
\begin{aligned}
\dot{F}(w ; x, \theta) & =\lim _{t \searrow 0} \frac{1}{t}(w(x)-w(x-t \theta)) \\
& =\nabla w(x) \theta \in \mathbb{R}^{M \times 1} .
\end{aligned}
$$

Thus, from (5.7) it follows that

$$
\lim _{\varepsilon \searrow 0} \mathcal{R}_{\left[\mathrm{d}_{1}\right], \varepsilon}(w) \approx \int_{\Omega} \underbrace{(\nabla w(x))^{\mathrm{T}} \nabla w(x)}_{\|\nabla w(x)\|_{\mathbb{R}^{M}}^{2}} \mathrm{~d} x .
$$

This is exactly the identity derived in Bourgain et al. [14].

From these considerations we can view $\lim _{\varepsilon \backslash 0} \mathcal{R}_{\left[\mathrm{d}_{1}\right], \varepsilon}$ as functionals, which generalize Sobolev and BV semi-norms to functions with values on manifolds.

\subsection{A Conjecture on Sobolev Semi-norms}

Starting point for this conjecture is Eq. 2.8. We will write $\Omega, M$ and $p$ instead of $\Omega_{1}, M_{1}$ and $p_{1}$.

- In the case $l=0, k=N, 0<s<1$ and $\mathrm{d}_{1}(w(x), w(y))=\|w(x)-w(y)\|_{\mathbb{R}^{M}}$ the functional $\mathcal{R}_{\left[\mathrm{d}_{1}\right]}$ from Eq. 2.8 simplifies to the $p$-th power of the Sobolev semi-norm and reads

$$
\int_{\Omega \times \Omega} \frac{\|w(x)-w(y)\|_{\mathbb{R}^{M}}^{p}}{\|x-y\|_{\mathbb{R}^{N}}^{N+p s}} \mathrm{~d}(x, y) .
$$

For a recent survey on fractional Sobolev spaces, see [25].

- On the other hand, when we choose $k=0, l=1$ and $\mathrm{d}_{1}(w(x), w(y))=\|w(x)-w(y)\|_{\mathbb{R}^{M}}$, then $\mathcal{R}_{\left[\mathrm{d}_{1}\right]}$ from Eq. 2.8 reads (note $\rho=\rho_{\varepsilon}$ by simplification of notation):

$$
\int_{\Omega \times \Omega} \frac{\|w(x)-w(y)\|_{\mathbb{R}^{M}}^{p}}{\|x-y\|_{\mathbb{R}^{N}}^{p s}} \rho_{\varepsilon}(x-y) \mathrm{d}(x, y) .
$$


- Therefore, in analogy to what we know for $s=1$ from [14], we conjecture that

$$
\begin{aligned}
& \lim _{\varepsilon \rightarrow 0} \int_{\Omega \times \Omega} \frac{\|w(x)-w(y)\|_{\mathbb{R}^{M}}^{p}}{\|x-y\|_{\mathbb{R}^{N}}^{p s}} \rho_{\varepsilon}(x-y) \mathrm{d}(x, y) \\
& =C \int_{\Omega \times \Omega} \frac{\|w(x)-w(y)\|_{\mathbb{R}^{M}}^{p}}{\|x-y\|_{\mathbb{R}^{N}}^{N+p s}} \mathrm{~d}(x, y) .
\end{aligned}
$$

The form Eq. 5.11 is numerically preferable to the standard Sobolev semi-norm Eq. 5.9, because $\rho=\rho_{\varepsilon}$ and thus the integral kernel has compact support.

\section{Numerical Examples}

In this section we present some numerical examples for denoising and inpainting of functions with values on the circle $\mathbb{S}^{1}$. Functions with values on a sphere have already been investigated very diligently (see, for instance, [13] out of series of publications of these authors). Therefore, we review some of their results first.

\section{$6.1 \mathbb{S}^{1}$-Valued Data}

Let $\emptyset \neq \Omega \subset \mathbb{R}$ or $\mathbb{R}^{2}$ be a bounded and simply connected open set with Lipschitz boundary. In [13] the question was considered when $w \in W^{s, p}\left(\Omega, \mathbb{S}^{1}\right)$ can be represented by some function $u \in W^{s, p}(\Omega, \mathbb{R})$ satisfying

$\Phi(u):=\mathrm{e}^{i u}=w$.

That is, the function $u$ is a lifting of $w$.

Lemma 6.1 ([13])

- Let $\Omega \subset \mathbb{R}, 0<s<\infty, 1<p<\infty$. Then for all $w \in W^{s, p}\left(\Omega, \mathbb{S}^{1}\right)$ there exists $u \in W^{s, p}(\Omega, \mathbb{R})$ satisfying $E q$. 6.1.

- Let $\Omega \subset \mathbb{R}^{N}, N \geq 2,0<s<1,1<p<\infty$. Moreover, let $s p<1$ or $s p \geq N$, then for all $w \in W^{s, p}\left(\Omega, \mathbb{S}^{1}\right)$ there exists $u \in W^{s, p}(\Omega, \mathbb{R})$ satisfying $E q .6 .1$.

If $s p \in[1, N)$, then there exist functions $w \in W^{s, p}\left(\Omega, \mathbb{S}^{1}\right)$ such that Eq. 6.1 does not hold with any function $u \in$ $W^{s, p}(\Omega, \mathbb{R})$.

For

$\mathrm{d}_{\mathbb{S}^{1}}(a, b):=\arccos \left(a^{\mathrm{T}} b\right), \quad a, b \in \mathbb{S}^{1}$, we consider the functional (note that by simplification of notation below $\rho=\rho_{\varepsilon}$ denotes a mollifier)

$\mathcal{R}_{\left[\mathrm{d}_{\mathbb{S}^{1}}\right]}(w)=\int_{\Omega \times \Omega} \frac{\mathrm{d}_{\mathbb{S}^{1}}^{p}(w(x), w(y))}{\|x-y\|_{\mathbb{R}^{N}}^{k+p s}} \rho^{l}(x-y) \mathrm{d}(x, y)$,

on $w \in W^{s, p}\left(\Omega, \mathbb{S}^{1}\right)$, in accordance to Eq. 2.8.

Writing $w=\Phi(u)$ as in Eq. 6.1, we get the lifted functional

$\mathcal{R}_{\left[\mathrm{d}_{\left.\mathbb{S}^{1}\right]}^{\Phi}\right.}(u):=\int_{\Omega \times \Omega} \frac{\mathrm{d}_{\mathbb{S}^{1}}^{p}(\Phi(u)(x), \Phi(u)(y))}{\|x-y\|_{\mathbb{R}^{N}}^{k+p s}} \rho^{l}(x-y) \mathrm{d}(x, y)$,

over the space $W^{s, p}(\Omega, \mathbb{R})$.

Remark 6.2 - We note that in the case $k=0, s=1$ and $l=1$ these integrals correspond with the ones considered in Bourgain et al. [14] for functions with values on $\mathbb{S}^{1}$.

- If we choose $k=N, s=1$ and $l=0$, then this corresponds with Sobolev semi-norms on manifolds.

- Let $\varepsilon>0$ fixed (that is, we consider neither a standard Sobolev regularization nor the limiting case $\varepsilon \rightarrow 0$ as in [14]). In this case we have proven coercivity of the functional $\mathcal{F}: W^{s, p}\left(\Omega, \mathbb{S}^{1}\right) \rightarrow[0, \infty), 0<s<1$, only with the following regularization functional, cf. Example 3.7 and Example 3.10:

$$
\int_{\Omega \times \Omega} \frac{\mathrm{d}_{\mathbb{S}^{1}}^{p}(w(x), w(y))}{\|x-y\|_{\mathbb{R}^{N}}^{N+p s}} \rho_{\varepsilon}(x-y) \mathrm{d}(x, y) .
$$

We summarize a few results: The first lemma follows from elementary calculations:

Lemma $6.3 \mathrm{~d}_{\mathbb{S}^{1}}$ and $\left.\mathrm{d}_{\mathbb{R}^{2}}\right|_{\mathbb{S}^{1} \times \mathbb{S}^{1}}$ are equivalent.

Lemma 6.4 Let $u \in W^{s, p}(\Omega, \mathbb{R})$. Then $\Phi(u) \in W^{s, p}\left(\Omega, \mathbb{S}^{1}\right)$.

Proof This follows directly from the inequality $\left\|\mathrm{e}^{i a}-\mathrm{e}^{i b}\right\| \leq$ $\|a-b\|$ for all $a, b \in \mathbb{R}$.

Below we show that $\mathcal{R}_{\left[\mathrm{d}_{\mathbb{S} 1}\right]}^{\Phi}$ is finite on $W^{s, p}(\Omega, \mathbb{R})$.

Lemma $6.5 \mathcal{R}_{\left[\mathrm{d}_{\mathbb{S} 1}\right]}^{\Phi}$ maps $W^{s, p}(\Omega, \mathbb{R})$ into $[0, \infty)$ (i.e., does not attain the value $+\infty$ ).

Proof Let $u \in W^{s, p}(\Omega, \mathbb{R})$. Then by Lemma 6.4 we have that $\Phi(u) \in W^{s, p}\left(\Omega, \mathbb{S}^{1}\right)$. Therefore, from Lemma 6.3 and Proposition 2.13 item (ii) it follows that $\mathcal{R}_{\left[\mathrm{d}_{\mathbb{S} 1}\right]}(\Phi(u))<\infty$. Hence, by definition, $\mathcal{R}_{\left[\mathrm{d}_{\mathbb{S} 1}\right]}^{\Phi}(u)<\infty$. 


\subsection{Setting of Numerical Examples}

In all numerical examples presented, we use a simplified setting with

$M_{1}=M_{2}=: M, K_{1}=K_{2}=: \mathbb{S}^{1}$,

$p_{1}=p_{2}=: p, k=N, l=1$,

$\Omega_{1}=\Omega_{2}=: \Omega$ when considering image denoising, $\Omega_{1}=\Omega$,

$\Omega_{2}=\Omega \backslash D$ when considering image inpainting, and

$W\left(\Omega, \mathbb{S}^{1}\right)=W^{s, p}\left(\Omega, \mathbb{S}^{1}\right)$.

As a particular mollifier, we use $\rho_{\varepsilon}$ (see Example 2.2), which is defined via the one-dimensional normal distribution $\hat{\rho}(x)=\frac{1}{\sqrt{\pi}} \mathrm{e}^{-x^{2}}$.

\section{Regularization Functionals}

Let $\mathcal{R}_{\left[\mathrm{d}_{\mathbb{1} 1}\right]}$ and $\mathcal{R}_{\left[\mathrm{d}_{\mathbb{S} 1}\right]}^{\Phi}$ be as defined in Eqs. 6.3 and 6.4, respectively. In what follows, we consider the following regularization functional

$\mathcal{F}_{\alpha}^{v^{\delta}}\left[\mathrm{d}_{\mathbb{S}^{1}}\right](w):=\int_{\Omega} \mathrm{d}_{\mathbb{S}^{1}}^{p}\left(\mathrm{~F}(w)(x), v^{\delta}(x)\right) \mathrm{d} x+\alpha \mathcal{R}_{\left[\mathrm{d}_{\mathbb{S}^{1}}\right]}(w)$,

on $W^{s, p}\left(\Omega, \mathbb{S}^{1}\right)$ and the lifted variant

$$
\tilde{\mathcal{F}}_{\alpha}^{v^{\delta}}\left[\mathrm{d}_{\mathbb{S} 1}\right](u):=\int_{\Omega} \mathrm{d}_{\mathbb{S}^{1}}^{p}\left(\mathrm{~F}(\Phi(u))(x), v^{\delta}(x)\right) \mathrm{d} x+\alpha \mathcal{R}_{\left[\mathrm{d}_{\mathbb{S}^{1}}\right]}^{\Phi}(u)
$$

over the space $W^{s, p}(\Omega, \mathbb{R})$ (as in Sect. 6.1), where $\Phi$ is defined as in (6.1). Note that $\tilde{\mathcal{F}}=\mathcal{F} \circ \Phi$.

Lemma 6.6 Let $\emptyset \neq \Omega \subset \mathbb{R}$ or $\mathbb{R}^{2}$ be a bounded and simply connected open set with Lipschitz boundary. Let $1<p<\infty$ and $s \in(0,1)$. If $N=2$ assume that $s p<1$ or $s p \geq 2$. Moreover, let Assumption 3.2 and Assumption 2.10 be satisfied. Then the mapping $\tilde{\mathcal{F}}_{\alpha}^{v^{\delta}}\left[\mathrm{d}_{\mathbb{S}^{1}}\right]: W^{s, p}(\Omega, \mathbb{R}) \rightarrow[0, \infty)$ attains a minimizer.

Proof Let $u \in W^{s, p}(\Omega, \mathbb{R})$. Then by Lemma 6.4 we have that $w:=\Phi(u) \in W^{s, p}\left(\Omega, \mathbb{S}^{1}\right)$. As arguing as in the proof of Lemma 6.5, we see that $\tilde{\mathcal{F}}_{\alpha}^{v^{\delta}}\left[\mathrm{d}_{\mathbb{S}^{1}}\right](u)<\infty$.

Since we assume that Assumption 3.2 is satisfied, we get that $\mathcal{F}_{\alpha}^{v^{\delta}}\left[\mathrm{d}_{\mathbb{S}^{1}}\right](w)$ attains a minimizer $w^{*} \in W^{s, p}\left(\Omega, \mathbb{S}^{1}\right)$. It follows from Lemma 6.1 that there exists a function $u^{*} \in$ $W^{s, p}(\Omega, \mathbb{R})$ that can be lifted to $w^{*}$, i.e., $w^{*}=\Phi\left(u^{*}\right)$. Then $u^{*}$ is a minimizer of (6.6) by definition of $\tilde{\mathcal{F}}$ and $\Phi$.

\subsection{Numerical Minimization}

In our concrete examples, we will consider two different operators F. For numerical minimization we consider the functional from Eq. 6.6 in a discretized setting. For this purpose, we approximate the functions $u \in W^{s, p}(\Omega, \mathbb{R})$, $0<s<1,1<p<\infty$ by quadratic B-spline functions and optimize with respect to the coefficients. We remark that this approximation is continuous and thus that sharp edges correspond to very steep slopes.

The noisy data $u^{\delta}$ are obtained by adding Gaussian white noise with variance $\sigma^{2}$ to the approximation or the discretized approximation of $u$.

We apply a simple Gradient Descent scheme with fixed step length implemented in MATLAB.

\subsection{Denoising of $\mathbb{S}^{1}$-Valued Functions: The InSAR Problem}

In this case the operator $\mathrm{F}: W^{s, p}\left(\Omega, \mathbb{S}^{1}\right) \rightarrow L^{p}\left(\Omega, \mathbb{S}^{1}\right)$ is the inclusion operator. It is norm-coercive in the sense of Eq. 3.4 and hence Assumption 3.2 is fulfilled. For $\emptyset \neq$ $\Omega \subset \mathbb{R}$ or $\mathbb{R}^{2}$ a bounded and simply connected open set, $1<p<\infty$ and $s \in(0,1)$ such that additionally $s p<1$ or $s p \geq 2$ if $N=2$ we can apply Lemma 6.6 which ensures that the lifted functional $\tilde{\mathcal{F}}_{\alpha}^{\delta}\left[\mathrm{d}_{\mathbb{S}^{1}}\right]: W^{s, p}(\Omega, \mathbb{R}) \rightarrow[0, \infty)$ attains a minimizer $u \in W^{s, p}(\Omega, \mathbb{R})$.

In the examples we will just consider the continuous approximation again denoted by $u$.

\section{One-Dimensional Test Case}

Let $\Omega=(0,1)$ and consider the signal $u: \Omega \rightarrow[0,2 \pi)$ representing the angle of a cyclic signal.

For the discrete approximation shown in Fig. 2a, the domain $\Omega$ is sampled equally at 100 points. $u$ is affected by an additive white Gaussian noise with $\sigma=0.1$ to obtain the noisy signal which is colored in blue in Fig. 2a.

In this experiment we show the influence of the parameters $s$ and $p$. In all cases the choice of the regularization parameter $\alpha$ is 0.19 and $\varepsilon=0.01$.

The red signal in Fig. $2 \mathrm{~b}$ is obtained by choosing $s=$ 0.1 and $p=1.1$. We see that the periodicity of the signal is handled correctly and that there is nearly no staircasing. In Fig. $2 c$ the parameter $s$ is changed from 0.1 to 0.6. The value of the parameter $p$ stays fixed. Increasing of $s$ leads the signal to be more smooth. We can observe an even stronger similar effect when increasing $p$ (here from 1.1 to 2 ) and letting $s$ fixed, see Fig. 2d. This fits the expectation since $s$ only appears once in the denominator of the regularizer. At a jump, increasing of $s$ leads thus to an increasing of the 


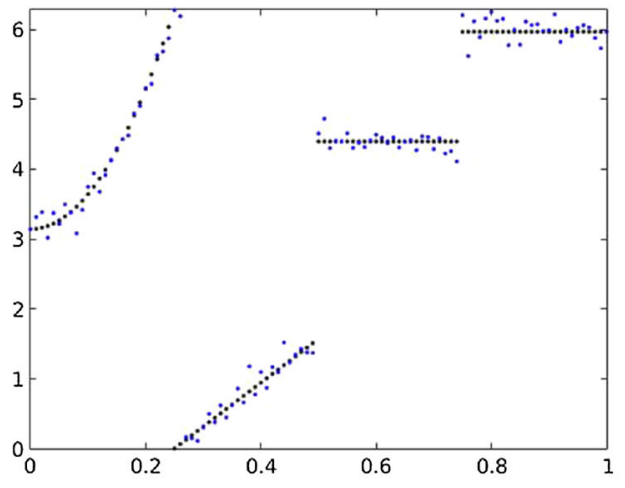

(a) Original and noisy data

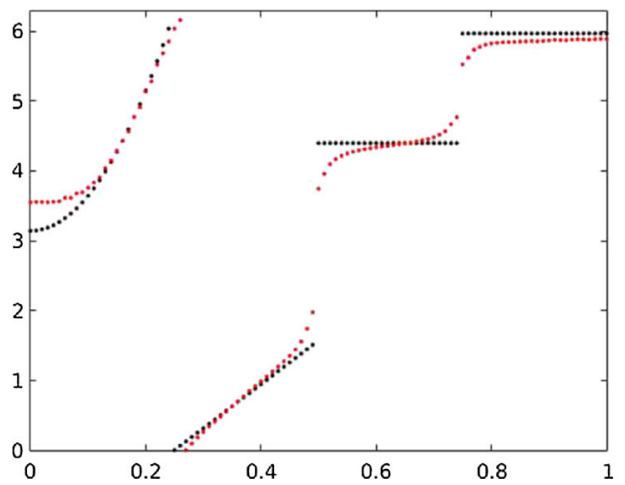

(c) Increasing of $s$

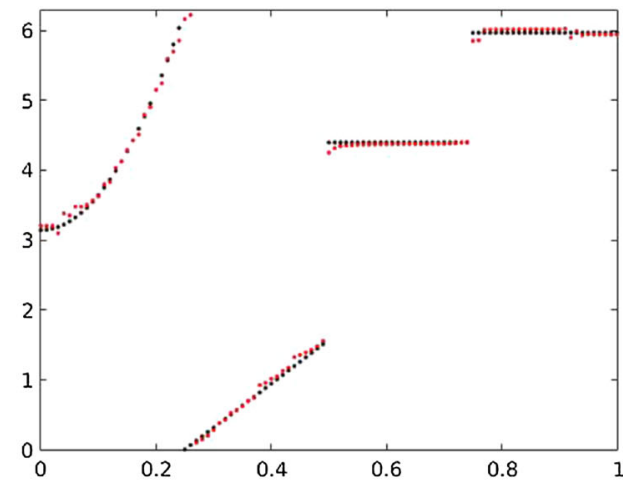

(b) Denoised data

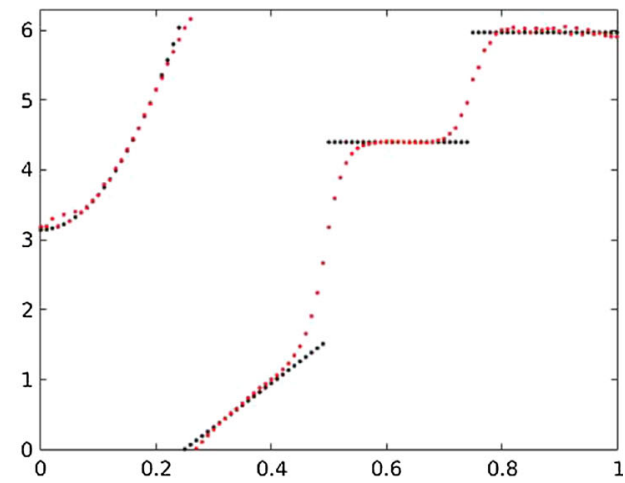

(d) Increasing of $p$

Fig. 2 Function on $\mathbb{S}^{1}$ represented in [0,2 $\pi$ ): Left to right, top to bottom: original data (black) and noisy data (blue) with 100 data points. Denoised data (red) where we chose $s=0.1, p=1.1, \alpha=0.19$. Denoised data with $s=0.6, p=1.1, \alpha=0.19$ resp. $s=0.1, p=2, \alpha=0.19$ (Color figure online)

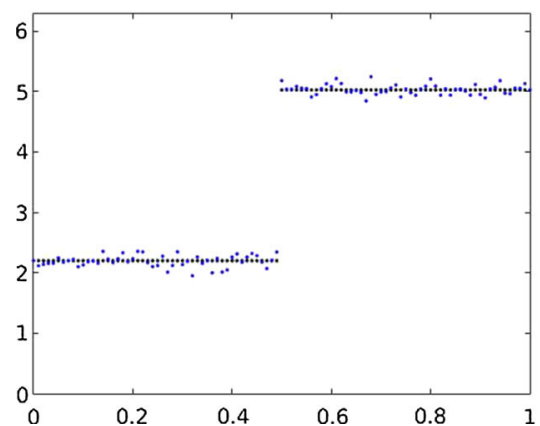

(a) Original and noisy data

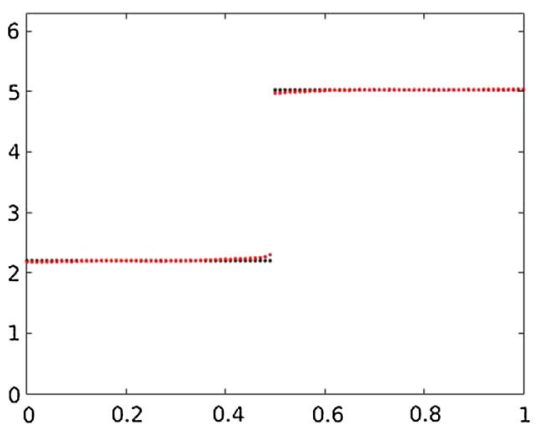

(b) $s=0.9, p=1.01$

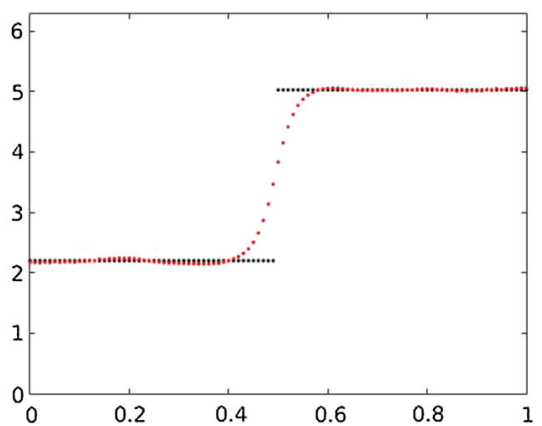

(c) $s=0.001, p=2$

Fig. 3 Left to right: original data (black) and noisy data (blue) sampled at 100 data points. Denoised data (red) where we chose $s=0.9, p=$ $1.01, \alpha=0.03$. Denoised data with $s=0.001, p=2, \alpha=0.9$ (Color figure online)

regularization term. The parameter $p$ appears twice in the regularizer. Huge jumps are hence weighted even more.

In Fig. 3a we considered a simple signal with a single huge jump. Again it is described by the angular value. We proceeded as above to obtain the approximated discrete original data (black) and noisy signal with $\sigma=0.1$ (blue). We chose again $\varepsilon=0.01$.

As we have seen above, increasing of $s$ leads to a more smooth signal. This effect can be compensated by choosing a rather small value of $p$, i.e., $p \approx 1$. In Fig. $3 b$ the value of $s$ is 0.9 . We see that it is still possible to reconstruct jumps by choosing, e.g., $p=1.01$.

Moreover, we have seen that increasing of $p$ leads to an even more smooth signal. In Fig. $3 \mathrm{c}$ we choose a quite large value of $p, p=2$ and a rather small value of $s, s=0.001$. Even for this very simple signal, it was not possible to get sharp edges. This is due to the fact that the parameter $p$ 


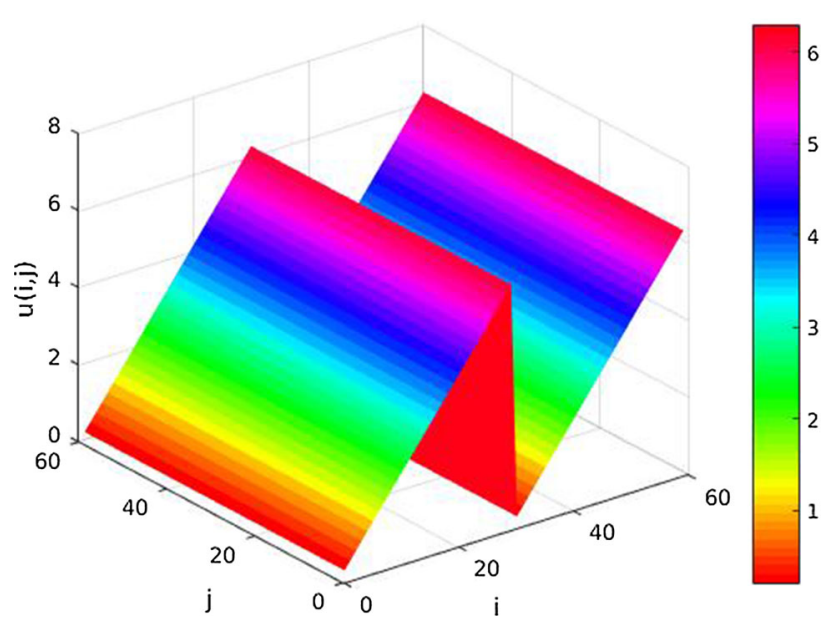

Fig. 4 The function $u$ evaluated on the discrete grid

(but not $s$ ) additionally weights the height of jumps in the regularizing term.

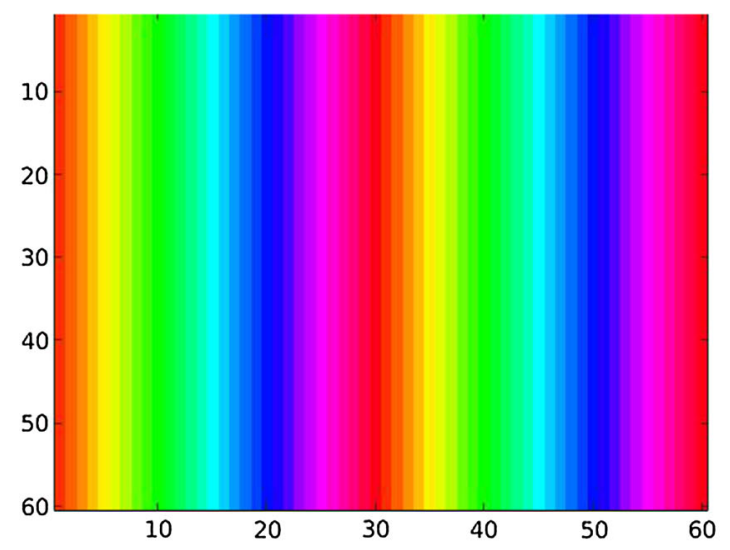

(a) Original data

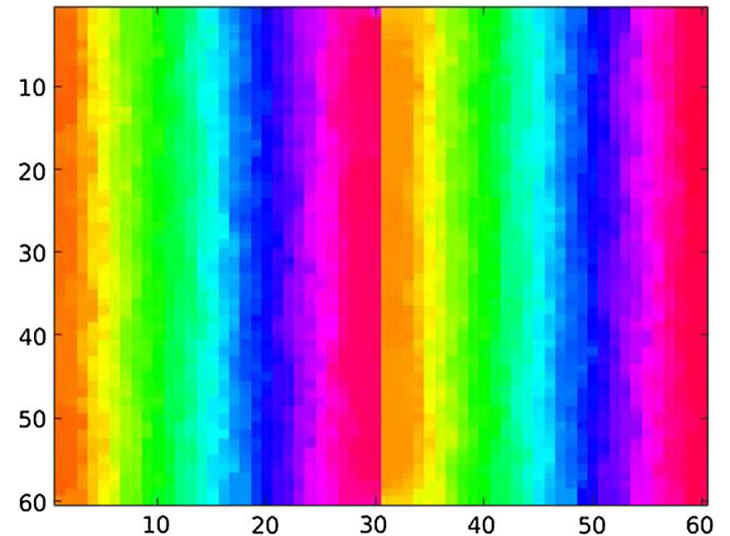

(c) TV-denoised data

\section{Denoising of a $\mathbb{S}^{1}$-Valued Image}

Our next example concerned a two-dimensional $\mathbb{S}^{1}$-valued image represented by the corresponding angular values. We remark that in this case where $N=2$ the existence of such a representation is always guaranteed in the cases where $s p<$ 1 or $s p \geq 2$, see Lemma 6.1 .

The domain $\Omega$ is sampled into $60 \times 60$ data points and can be considered as discrete grid, $\{1, \ldots, 60\} \times\{1, \ldots, 60\}$. The B-spline approximation evaluated at that grid is given by

$u(i, j)=u(i, 0):=4 \pi \frac{i}{60} \bmod 2 \pi, \quad i, j \in\{1, \ldots, 60\}$.

The function $u$ is shown in Fig. 4. We used the hsv colormap provided in MATLAB transferred to the interval $[0,2 \pi]$.

This experiment shows the difference of our regularizer respecting the periodicity of the data in contrast to the classical total variation regularizer. The classical TV minimization is solved using a fixed point iteration ([45]); for the method see also [60].

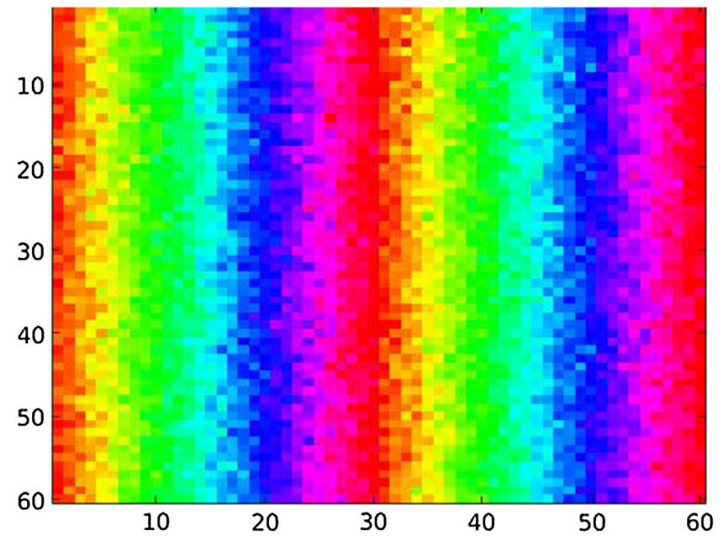

(b) Noisy data

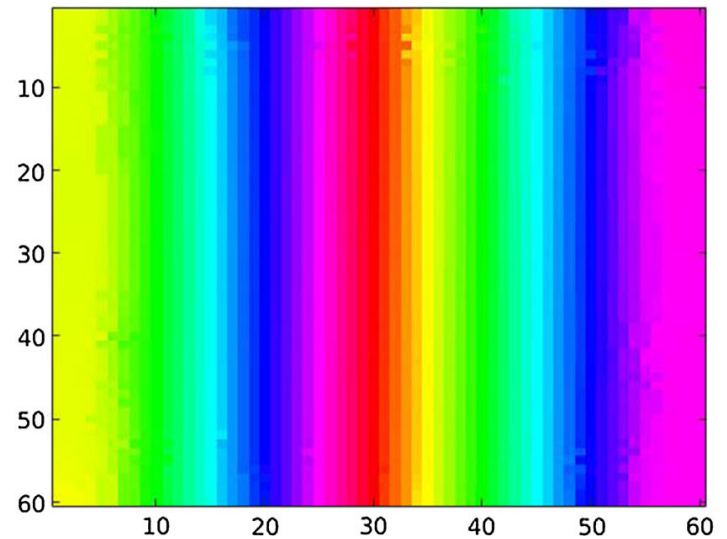

(d) Denoised data

Fig. 5 Left to right, top to bottom: original and noisy data of an $60 \times 60$ image. TV-denoised data using a fixed point iteration method. Denoised data where we chose $s=0.9, p=1.1, \alpha=1,400$ steps 


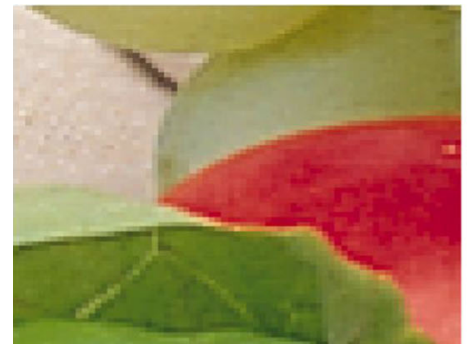

(a) Original $R G B$ image

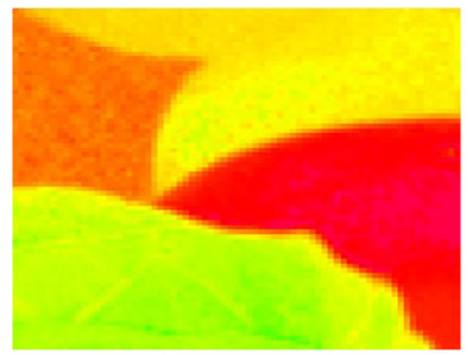

(b) Hue component represented in color, which represent function values on $\mathbb{S}^{1}$

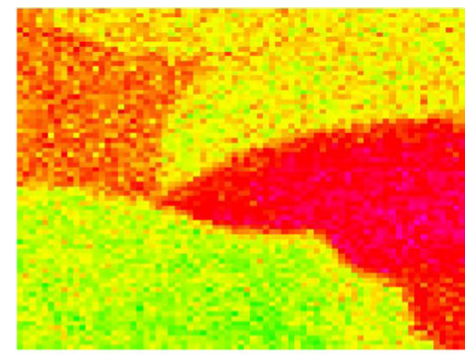

(c) Noisy hue value - again representing function values on $\mathbb{S}^{1}$

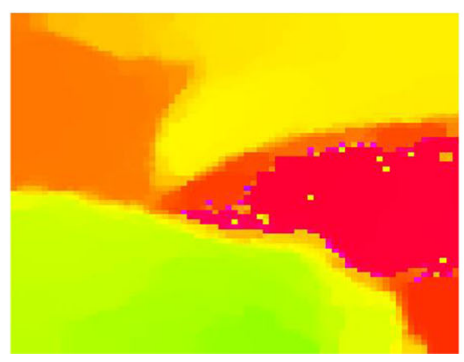

(d) $T V$-denoised data

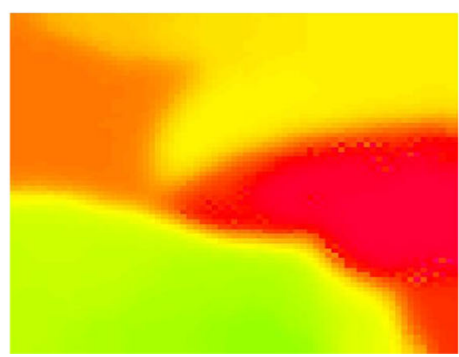

(e) Denoised data

Fig. 6 Left to right, top to bottom: original RGB image and its hue component. Noisy hue data with $\sigma^{2}=0.001$. TV minimization is done using an iterative approach. It is serving as starting point for the GD minimization. Denoised data with $s=0.49, p=2, \alpha=2,500$ steps

In Fig. 5a the function $u$ can be seen from the top, i.e., the axes correspond to the $i$ resp. $j$ axis in Fig. 4. The noisy data are obtained by adding white Gaussian noise with $\sigma=$ $\sqrt{0.001}$ using the built-in function imnois se in MATLAB. It is shown in Fig. 5b. We choose as parameters $s=0.9, p=$ $1.1, \alpha=1$, and $\varepsilon=0.01$. We observe significant noise reduction in both cases. However, only in Fig. 5d the color transitions are handled correctly. This is due to the fact that our regularizer respects the periodicity, i.e., for the functional there is no jump in Fig. 4 since 0 and $2 \pi$ are identified. Using the classical TV regularizer, the values 0 and $2 \pi$ are not identified and have a distance of $2 \pi$. Hence, in the TVdenoised image there is a sharp edge in the middle of the image, see Fig. 5c.

\section{Hue Denoising}

The HSV color space is shorthand for Hue, Saturation, Value (of brightness). The hue value of a color image is $\mathbb{S}^{1}$-valued, while saturation and value of brightness are real-valued. Representing colors in this space better match the human perception than representing colors in the RGB space.

In Fig. 6a we see a part of size $70 \times 70$ of the RGB image "fruits" (https://homepages.cae.wisc.edu/ ece533/images/).
The corresponding hue data are shown in Fig. 6b, where we used again the colormap HSV, cf. Fig. 4. Each pixel value lies, after transformation, in the interval $[0,2 \pi)$ and represents the angular value. Gaussian white noise with $\sigma=\sqrt{0.001}$ is added to obtain a noisy image, see Fig. 6c.

To obtain the denoised image, in Fig. 6d we again used the same fixed point iteration, cf. [45], as before.

We see that the denoised image suffers from artifacts due to the non-consideration of periodicity. The pixel values in the middle of the apple (the red object in the original image) are close to $2 \pi$ while those close to the border are nearly 0 , meaning they have a distance of around $2 \pi$.

We use this TV-denoised image as starting image to perform the minimization of our energy functional. As parameters we choose $s=0.49, p=2, \alpha=2, \varepsilon=0.006$.

Since the cyclic structure is respected, the disturbing artifacts in image in Fig. 6d are removed correctly. The edges are smoothed due to the high value of $p$, see Fig. 6 e.

\section{$6.5 \mathbb{S}^{1}$-Valued Image Inpainting}

In this case the operator $\mathrm{F}: W^{s, p}\left(\Omega, \mathbb{S}^{1}\right) \rightarrow L^{p}\left(\Omega, \mathbb{S}^{1}\right)$ is the inpainting operator, i.e.,

$\mathrm{F}(w)=\chi_{\Omega \backslash D}(w)$, 


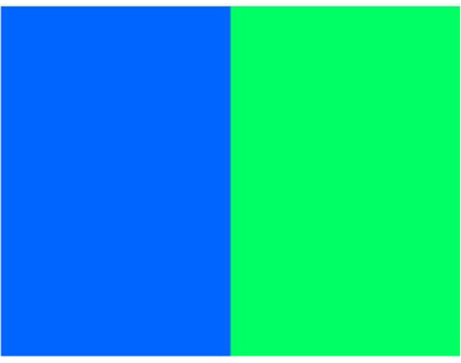

(a) Original image

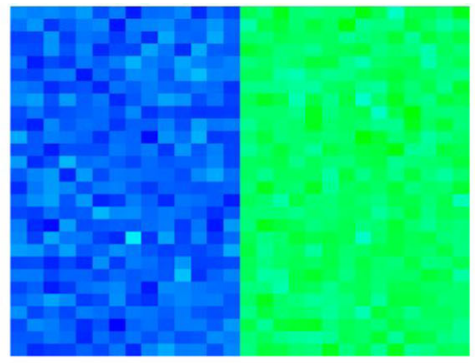

(b) Noisy image

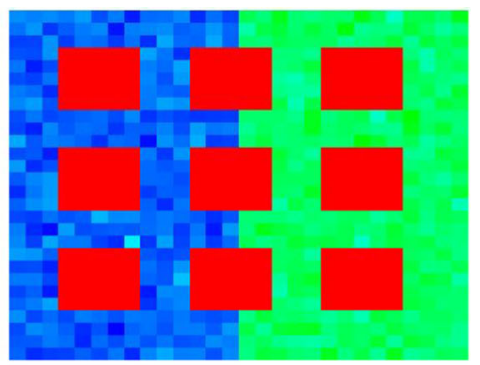

(c) Noisy masked image

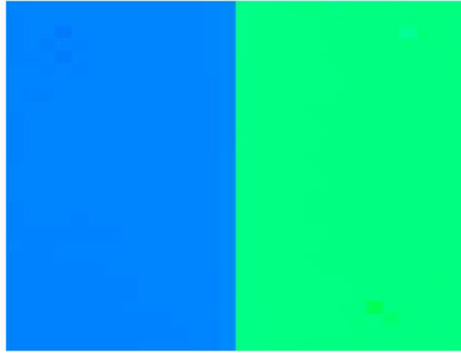

(d) Reconstructed image

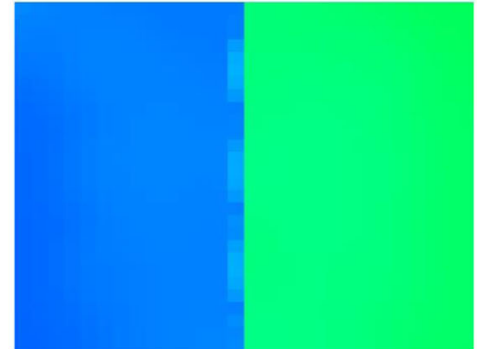

(e) $T V$-reconstructed image

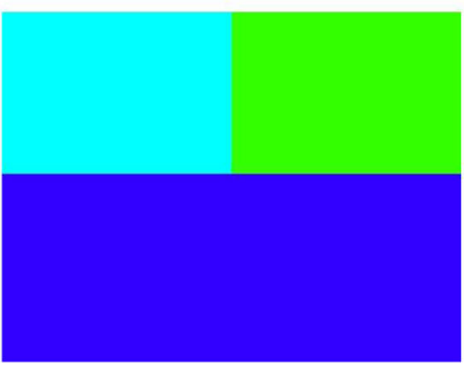

(f) Original image

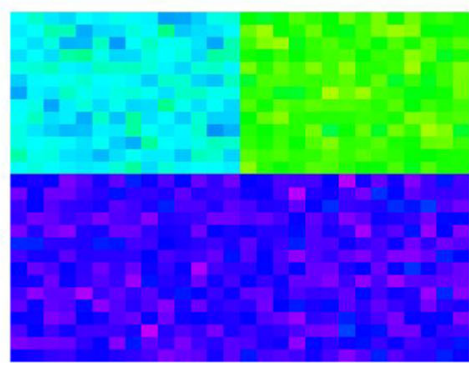

(g) Noisy image

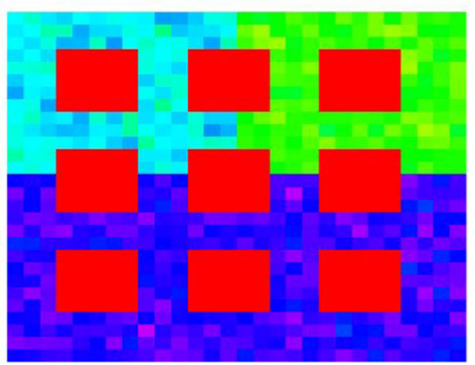

(h) Noisy masked image

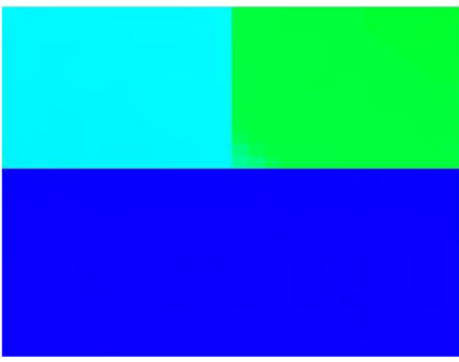

(i) Reconstructed image

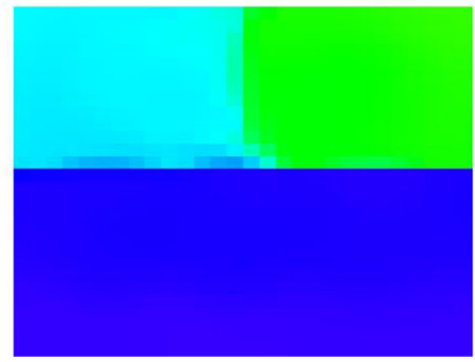

(j) $T V$-reconstructed image
Fig. 7 Left to right. Top to bottom: original image and the noisy data with $\sigma^{2}=0.001$. Noisy image with masking filter and denoised data with $s=0.3, p=1.01, \alpha=0.3,6000$ steps. TV-denoised data. Orig- inal image and the noisy data with $\sigma^{2}=0.001$. Noisy image with masking filter and denoised data with $s=0.4, p=1.01, \alpha=0.4$, 10000 steps. TV-denoised image 


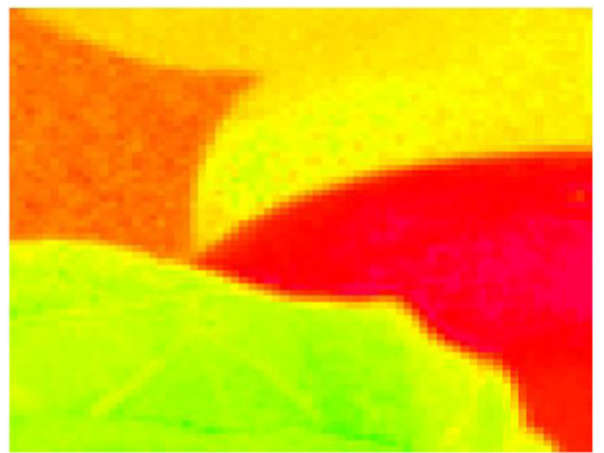

(a) Hue component

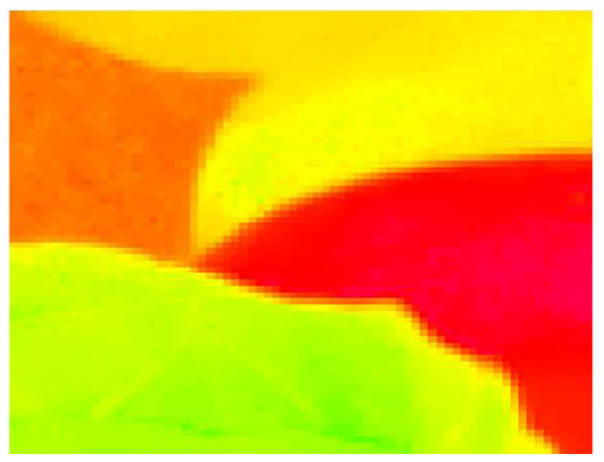

(c) TV-reconstructed image

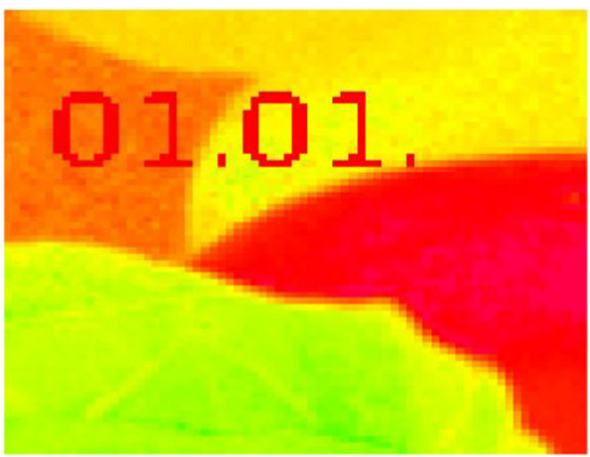

(b) Image with masked region

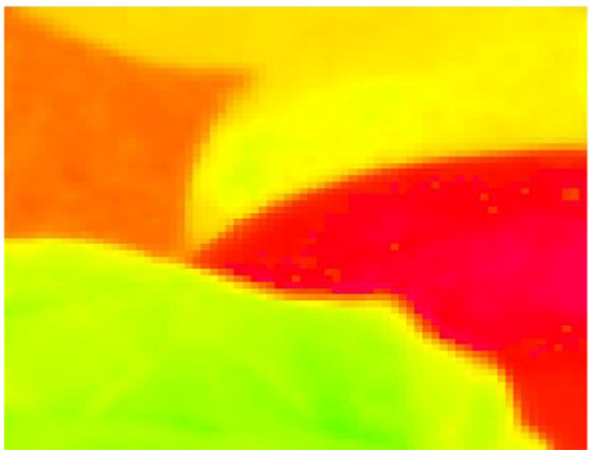

(d) Reconstructed image

Fig. 8 Left to right, top to bottom: original image and image with masked region. Reconstructed image with parameters $p=1.1, s=0.1, \alpha=2$ and $\varepsilon=0.006,2000$ steps. TV-reconstructed image

where $D \subseteq \Omega$ is the area to be inpainted.

We consider the functional

$$
\begin{aligned}
\mathcal{F}_{\alpha}^{\delta}\left[\mathrm{d}_{\mathbb{S} 1}\right](w):= & \int_{\Omega \backslash D} \mathrm{~d}_{\mathbb{S}^{1}}^{p}\left(w(x), v^{\delta}(x)\right) \mathrm{d} x \\
& \quad+\alpha \int_{\Omega \times \Omega} \frac{\mathrm{d}_{\mathbb{S}^{1}}^{p}(w(x), w(y))}{\|x-y\|_{\mathbb{R}^{2}}^{2+p s}} \rho_{\varepsilon}(x-y) \mathrm{d}(x, y),
\end{aligned}
$$

on $W^{s, p}\left(\Omega, \mathbb{S}^{1}\right)$.

According to Example 3.10, the functional $\mathcal{F}$ is coercive and Assumption 3.2 is satisfied. For $\emptyset \neq \Omega \subset \mathbb{R}$ or $\mathbb{R}^{2}$ a bounded and simply connected open set, $1<p<\infty$ and $s \in(0,1)$ such that additionally $s p<1$ or $s p \geq 2$ if $N=2$ Lemma 6.6 applies which ensures that there exists a minimizer $u \in W^{s, p}(\Omega, \mathbb{R})$ of the lifted functional $\tilde{\mathcal{F}}_{\alpha}^{v^{\delta}}\left[\mathrm{d}_{\mathbb{S} 1}\right]: W^{s, p}(\Omega, \mathbb{R}) \rightarrow[0, \infty) u \in W^{s, p}(\Omega, \mathbb{R})$

\section{Inpainting of a $\mathbb{S}^{1}$-Valued Image}

As a first inpainting test example, we consider two $\mathbb{S}^{1}$-valued images of size $28 \times 28$, see Fig. 7, represented by its angular values. In both cases the ground truth can be seen in Fig. 7a, f. We added Gaussian white noise with $\sigma=\sqrt{0.001}$ using the MATLAB build-in function imnoi se. The noisy images can be seen in Fig. 7b, g. The region $D$ consists of the nine red squares in Fig. 7c, h.

The reconstructed data are shown in Fig. 7d, i.

For the two-colored image, we used as parameters $\alpha=s=0.3, p=1.01$ and $\varepsilon=0.05$. We see that the reconstructed edge appears sharp. The unknown squares, which are completely surrounded by one color, are inpainted perfectly. The blue and green color changed slightly.

As parameters for the three-colored image, we used $\alpha=$ $s=0.4, p=1.01$ and $\varepsilon=0.05$. Here again the unknown regions lying entirely in one color are inpainted perfectly. The edges are preserved. Just the corner in the middle of the image is slightly smoothed.

In Fig. 7e, $\mathrm{j}$ the TV-reconstructed data are shown. The underlying algorithm ([31]) uses the split Bregman method (see [36]).

In Fig. 7e the edge is not completely sharp. There are some lighter parts on the blue side. This can be caused by the fact that the unknown domain in this area is not exactly symmetric with respect to the edge. This is also the case in Fig. $7 \mathrm{j}$ where we observe the same effect. 
Unknown squares lying entirely in one color are perfectly inpainted.

\section{Hue Inpainting}

As a last example, we consider again the hue component of the image "fruits", see Fig. 8a. The unknown region $D$ is the string 01.01 which is shown in Fig. 8b. As parameters we choose $p=1.1, s=0.1, \alpha=2$ and $\varepsilon=0.006$. We get the reconstructed image shown in Fig. 8c. The edges are preserved and the unknown area is restored quite well. This can be also observed in the TV-reconstructed image in Fig. 8d, using again the split Bregman method as before, cf. [31].

\subsection{Conclusion}

In this paper we developed a functional for regularization of functions with values in a set of vectors. The regularization functional is a derivative-free, nonlocal term, which is based on a characterization of Sobolev spaces of intensity data derived by Bourgain, Brézis, Mironescu and Dávila. Our objective has been to extend their double integral functionals in a natural way to functions with values in a set of vectors, in particular functions with values on an embedded manifold. These new integral representations are used for regularization on a subset of the (fractional) Sobolev space $W^{s, p}\left(\Omega, \mathbb{R}^{M}\right)$ and the space $B V\left(\Omega, \mathbb{R}^{M}\right)$, respectively. We presented numerical results for denoising of artificial InSAR data as well as an example of inpainting. Moreover, several conjectures are at hand on relations between double metric integral regularization functionals and single integral representations.

Acknowledgements Open access funding provided by Austrian Science Fund. We thank Peter Elbau for very helpful discussions and comments. MH and OS acknowledge support from the Austrian Science Fund (FWF) within the national research network Geometry and Simulation, Project S11704 (Variational Methods for Imaging on Manifolds). Moreover, OS is supported by the Austrian Science Fund (FWF), with SFB F68, Project F6807-N36 (Tomography with Uncertainties) and I3661-N27 (Novel Error Measures and Source Conditions of Regularization Methods for Inverse Problems).

Open Access This article is distributed under the terms of the Creative Commons Attribution 4.0 International License (http://creativecomm ons.org/licenses/by/4.0/), which permits unrestricted use, distribution, and reproduction in any medium, provided you give appropriate credit to the original author(s) and the source, provide a link to the Creative Commons license, and indicate if changes were made.

\section{References}

1. Adams, R.A.: Sobolev Spaces. Pure and Applied Mathematics, vol. 65. Academic Press, New York (1975)
2. Ambrosio, L., Fusco, N., Pallara, D.: Functions of Bounded Variation and Free Discontinuity Problems. Oxford Mathematical Monographs, p. xviii+434. Oxford University Press, New York (2000)

3. Aubert, G., Kornprobst, P.: Can the nonlocal characterization of Sobolev spaces by Bourgain et al. be useful for solving variational problems? SIAM J. Numer. Anal. 47(2), 844-860 (2009). https:// doi.org/10.1137/070696751

4. Bacák, M., Bergmann, R., Steidl, G., Weinmann, A.: A second order non-smooth variational model for restoring manifold-valued images. SIAM J. Sci. Comput. 38(1), A567-A597 (2016). https:// doi.org/10.1137/15M101988X

5. Bergmann, R., Chan, R.H., Hielscher, R., Persch, J., Steidl, G.: Restoration of manifold-valued images by half-quadratic minimization. Inverse Probl. Imaging 10(2), 281-304 (2016). https:// doi.org/10.3934/ipi.2016001

6. Bergmann, R., Fitschen, J.H., Persch, J., Steidl, G.: Priors with coupled first and second order differences for manifold-valued image processing. J. Math. Imaging Vis. 60, 1459-1481 (2018)

7. Bergmann, R., Laus, F., Steidl, G., Weinmann, A.: Second order differences of cyclic data and applications in variational denoising. SIAM J. Imaging Sci. 7(4), 2916-2953 (2014). https://doi.org/10. 1137/140969993

8. Bergmann, R., Persch, J., Steidl, G.: A parallel Douglas-Rachford algorithm for restoring images with values in symmetric Hadamard manifolds. SIAM J. Imaging Sci. 9(3), 901-937 (2016). https://doi. org/10.1137/15M1052858

9. Bergmann, R., Weinmann, A.: A second order TV-type approach for inpainting and denoising higher dimensional combined cyclic and vector space data. J. Math. Imaging Vis. 55(3), 401-427 (2016). https://doi.org/10.1007/s10851-015-0627-3

10. Bergmann, R., Weinmann, A.: Inpainting of cyclic data using first and second order differences. In: Tai, X.-C., Bae, E., Chan, T.F., Leung, S.Y., Lysaker, M. (eds.). EMMCVPR 2015, pp. 155168. Springer, Berlin (2015). https://doi.org/10.1007/978-3-31914612-6_12

11. Boulanger, J., Elbau, P., Pontow, C., Scherzer, O.: Non-local functionals for imaging. In: Bauschke, H.H., Burachik, R.S., Combettes, P.L., Elser, V., Luke, D.R., Wolkowicz, H. (eds.) Fixed-Point Algorithms for Inverse Problems in Science and Engineering. Springer Optimization and Its Applications, vol. 49, pp. 131-154. Springer, New York (2011). ISBN: 978-1-4419-9568-1. https://doi. org/10.1007/978-1-4419-9569-8

12. Bouman, C., Sauer, K.: A generalized Gaussian image model for edge-preserving MAP estimation. IEEE Trans. Image Process. 2(3), 296-310 (1993)

13. Bourgain, J., Brezis, H., Mironescu, P.: Lifting in Sobolev spaces. J. Anal. Math. 80, 37-86 (2000)

14. Bourgain, J., Brézis, H., Mironescu, P.: Another look at Sobolev spaces. In: Menaldi, J.L., Rofman, E., Sulem, A. (eds.) Optimal Control and Partial Differential Equations-Innovations and Applications: In honor of Professor Alain Bensoussan's 60th Anniversary, pp. 439-455. IOS Press, Amsterdam (2001)

15. Chambolle, A., Lions, P.-L.: Image recovery via total variation minimization and related problems. Numer. Math. 76(2), 167-188 (1997)

16. Cimrák, I., Melicher, V.: Mixed Tikhonov regularization in Banach spaces based on domain decomposition. Appl. Math. Computat. 218(23), 11583-11596 (2012). https://doi.org/10.1016/j.amc. 2012.05.042

17. Cremers, D., Koetter, S., Lellmann, J., Strekalovskiy, E.: Total variation regularization for functions with values in a manifold. In: IEEE international conference on computer vision, ICCV 2013, Sydney, Australia, 1-8 December 2013, pp. 2944-2951 (2013). https://doi.org/10.1109/ICCV.2013.366 
18. Cremers, D., Strekalovskiy, E.: Total cyclic variation and generalizations. J. Math. Imaging Vis. 47(3), 258-277 (2013)

19. Cremers, D., Strekalovskiy, E.: Total variation for cyclic structures: convex relaxation and efficient minimization. In: CVPR, pp. 19051911. IEEE Computer Society (2011). ISBN: 978-1-4577-0394-2

20. Dacorogna, B.: Direct Methods in the Calculus of Variations. Applied Mathematical Sciences, vol. 78. Springer, Berlin (1989)

21. Dacorogna, B.: Weak Continuity and Weak Lower Semicontinuity of Non-Linear Functionals. Lecture Notes in Mathematics, vol. 922. Springer, Berlin (1982)

22. Daubechies, I., Defrise, M., De Mol, C.: An iterative thresholding algorithm for linear inverse problems with a sparsity constraint. Commun. Pure Appl. Math. 57(11), 1413-1457 (2004)

23. Dávila, J.: On an open question about functions of bounded variation. Calc. Var. Partial Differ. Equ. 15(4), 519-527 (2002)

24. Demengel, F., Demengel, G.: Functional Spaces for the Theory of Elliptic Partial Differential Equations. Springer, New York (2007)

25. Di Nezza, E., Palatucci, G., Valdinoci, E.: Hitchhiker's guide to the fractional Sobolev spaces. Bull. Sci. Math. 136(5), 521-573 (2012). https://doi.org/10.1016/j.bulsci.2011.12.004

26. Eggermont, P.P.B.: Maximum entropy regularization for Fredholm integral equations of the first kind. SIAM J. Math. Anal. 24(6), 1557-1576 (1993)

27. Elstrodt, J.: Maß- und Integrationstheorie. Springer, Berlin (2002)

28. Engl, H.W., Landl, G.: Convergence rates for maximum entropy regularization. SIAM J. Numer. Anal. 30(5), 1509-1536 (1993)

29. Evans, L.C.: Partial Differential Equations. Graduate Studies in Mathematics, vol. 19. American Mathematical Society, Providence (2010)

30. Figalli, A., Villani, C.: Optimal transport and curvature. In: Ambrosio, L., Savaré, G. (eds.) Nonlinear PDE's and Applications. Lecture Notes in Mathematics, vol. 2028, pp. 171-217. Springer, Heidelberg (2011). https://doi.org/10.1007/978-3-642-21861-3_4

31. Getruer, P.: tvreg. From MathWorks-File Exchange

32. Giaquinta, M., Modica, G., Souček, J.: Variational problems for maps of bounded variation with values in S1. Calcul. Var. Partial Differ. Equ. 1(1), 87-121 (1993). https://doi.org/10.1007/ BF02163266

33. Giaquinta, M., Mucci, D.: Maps of bounded variation with values into a manifold: total variation and relaxed energy. Annali della Scuala Normale Superiore di Pisa. Classe di Scienze (5) 5(4), 483 548 (2006)

34. Giaquinta, M., Mucci, D.: The BV-energy of maps into a manifold: relaxation and density results. Int. J. Pure Appl. Math. 3(2), 513538 (2007)

35. Gilboa, G., Osher, S.: Nonlocal operators with applications to image processing. Multiscale Model. Simul. SIAM Interdiscip. J. 7(3), 1005-1028 (2008). https://doi.org/10.1137/070698592

36. Goldstein, T., Osher, S.: The split Bregman method for L1regularized problems. SIAM J. Imaging Sci. 2, 323-343 (2009)

37. Grohs, P., Sprecher, M.: Total Variation Regularization by Iteratively Reweighted Least Squares on Hadamard Spaces and the Sphere. Technical Report, 2014-39. Seminar for Applied Mathematics, ETH Zürich, Switzerland (2014)

38. Hadani, R., Singer, A.: Representation theoretic patterns in three dimensional cryo-electron microscopy I: the intrinsic reconstitution algorithm. Ann. Math. 174(2), 1219-1241 (2011)

39. Helliwell, C.A., Anderssen, R.S., Robertson, M., Finnegan, E.J.: How is FLC repression initiated by cold? Trends Plant Sci. 20, 76-82 (2015)

40. Kimmel, R., Sochen, N.A.: Orientation diffusion or how to comb a Porcupine. J. Vis. Commun. Image Represent. 13(1-2), 238-248 (2002). https://doi.org/10.1006/jvci.2001.0501

41. Kolehmainen, V., Lassas, M., Niinimäki, K., Siltanen, S.: Sparsitypromoting Bayesian inversion. Inverse Probl. 28(2), 025005, 28 (2012). https://doi.org/10.1088/0266-5611/28/2/025005
42. Lassas, M., Saksman, E., Siltanen, S.: Discretization-invariant Bayesian inversion and Besov space priors. J. Inverse Ill Posed Probl. 3(1), 87-122 (2009)

43. Laus, F., Nikolova, M., Persch, J., Steidl, G.: A nonlocal denoising algorithm for manifold-valued images using second order statistics. SIAM J. Imaging Sci. 10(1), 416-448 (2017)

44. Liu, J.G., Mason, J.: Image Processing and GIS for Remote Sensing, 2nd edn. Wiley, London (2016)

45. Loendahl, C., Magiera, P.: ROF Denoising Algorithm. From MathWorks-File Exchange

46. Lorenz, D., Trede, D.: Optimal convergence rates for Tikhonov regularization in Besov scales. Inverse Probl. 24(5), 055010 (2008). (14 pp)

47. Osher, S., Esedoglu, S.: Decomposition of Images by the anisotropic Rudin-Osher-Fatemi model. Commun. Pure Appl. Math. 57(12), 1609-1626 (2004)

48. Plataniotis, K., Venetsanopoulos, A.N.: Color Image Processing and Applications. Springer, Berlin (2000)

49. Ponce, A.: A new approach to Sobolev spaces and connections to $\Gamma$-convergence. Calcul. Var. Partial Differ. Equ. 19, 229-255 (2004)

50. Pöschl, C.: Tikhonov Regularization with General Residual Term. English. Ph.D. Thesis. University of Innsbruck, Innsbruck, Austria (2008)

51. Puttonen, E., Briese, C., Mandlburger, G., Wieser, M., Pfennigbauer, M., Zlinszky, A., Pfeifer, N.: Quantification of overnight movement of Birch (Betula pendula) branches and foliage with short interval terrestrial laser scanning. Front. Plant Sci. 7, 222 (2016)

52. Resmerita, E., Anderssen, R.S.: Joint additive Kullback-Leibler residual minimization and regularization for linear inverse problems. Math. Methods Appl. Sci. 30(13), 1527-1544 (2007)

53. Rocca, F., Prati, C., Ferretti, A.: An overview of SAR interferometry. In: 3rd ERS Symposiumm, Florence 97-Abstracts and Papers (1997)

54. Rudin, L.I., Osher, S., Fatemi, E.: Nonlinear total variation based noise removal algorithms. Phys. D Nonlinear Phenom. 60(1-4), 259-268 (1992)

55. Scherzer, O., Grasmair, M., Grossauer, H., Haltmeier, M., Lenzen, F.: Variational Methods in Imaging. Applied Mathematical Sciences, vol. 167. Springer, New York (2009). https://doi.org/10. 1007/978-0-387-69277-7

56. Scherzer, O., Weickert, J.: Relations between regularization and diffusion filtering. J. Math. Imaging Vis. 12(1), 43-63 (2000). https://doi.org/10.1023/A:1008344608808

57. Schuster, T., Kaltenbacher, B., Hofmann, B., Kazimierski, K.S.: Regularization Methods in Banach Spaces. Radon Series on Computational and Applied Mathematics, vol. 10, p. xii+283. De Gruyter, Berlin (2012). https://doi.org/10.1515/9783110255720

58. Singer, A., Shkolnisky, Y.: Viewing direction estimation in cryoEM using synchronization. SIAM J. Imaging Sci. 5(3), 1088-1110 (2012). https://doi.org/10.1137/120863642

59. Tikhonov, A.N., Arsenin, V.Y.: Solutions of Ill-Posed Problems. Wiley, Washington, DC (1977)

60. Vogel, C.R., Oman, M.E.: Iterative methods for total variation denoising. SIAM J. Sci. Comput. 17, 227-238 (1996)

61. Wang, L., Singer, A., Wen, Z.: Orientation determination of cryoEM images using least unsquared deviations. SIAM J. Imaging Sci. 6(4), 2450-2483 (2013). https://doi.org/10.1137/130916436

62. Weinmann, A., Demaret, L., Storath, M.: Total variation regularization for manifold-valued data. SIAM J. Imaging Sci. 7(4), 2226-2257 (2014) 
Publisher's Note Springer Nature remains neutral with regard to jurisdictional claims in published maps and institutional affiliations.

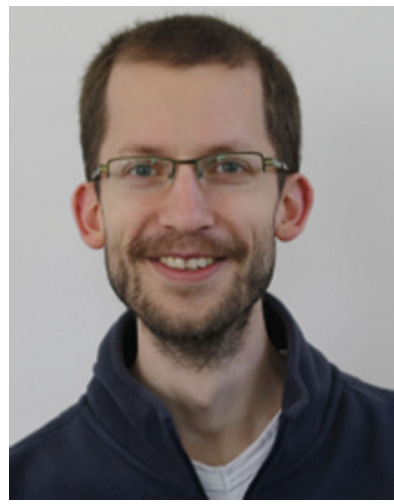

René Ciak received his diploma degree in mathematics from the University of Würzburg, Germany, and his Ph.D. from the University of Kaiserslautern. He is currently a postdoc at the Faculty of Mathematics at the University of Vienna, Austria.

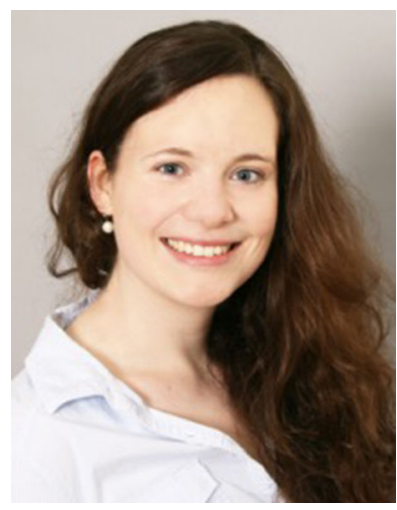

Melanie Melching received her masters degree in mathematics from the University of Bonn, Germany. She is currently a Ph.D. student at the Faculty of Mathematics at the University of Vienna, Austria.

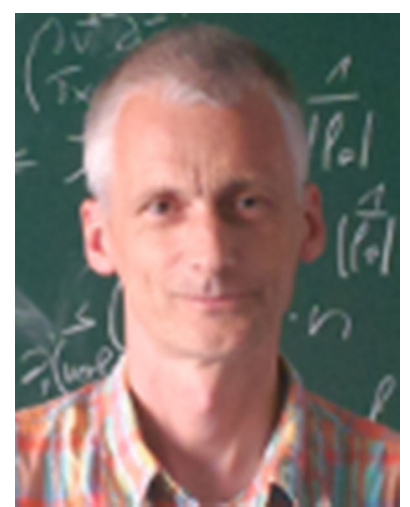

Otmar Scherzer received a Ph.D. in mathematics from the University of Linz, Austria, in 1990. From 1988 to 2000, he held the positions of Assistant Lecturer, Assistant Professor, and Associate Professor at the University of Linz. $\mathrm{He}$ held the position of Professor at the University of Munich, Germany, from 1999 to 2000, the University of Bayreuth, Germany, from 2000 to 2001, and the University of Innsbruck, Austria, from 2001 to 2009. Since 2009, he has been Professor of Computational Science, at the Faculty of Mathematics at the University of Vienna, Austria. He has also been with the Radon Institute of Applied Sciences, Linz, Austria, as a Scientific Group Leader of Mathematical Imaging and Inverse Problems. His research interests include image processing and inverse problems. 